\title{
Combined Pharmacological and Genetic Manipulations Unlock Unprecedented Temporal Elasticity and Reveal Phase-Specific Modulation of the Molecular Circadian Clock of the Mouse Suprachiasmatic Nucleus
}

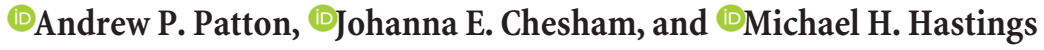 \\ Division of Neurobiology, Laboratory of Molecular Biology, Medical Research Council, Cambridge CB2 0QH, United Kingdom
}

The suprachiasmatic nucleus (SCN) is the master circadian oscillator encoding time-of-day information. SCN timekeeping is sustained by a cell-autonomous transcriptional-translational feedback loop, whereby expression of the Period and Cryptochrome genes is negatively regulated by their protein products. This loop in turn drives circadian oscillations in gene expression that direct SCN electrical activity and thence behavior. The robustness of SCN timekeeping is further enhanced by interneuronal, circuit-level coupling. The aim of this study was to combine pharmacological and genetic manipulations to push the SCN clockwork toward its limits and, by doing so, probe cell-autonomous and emergent, circuit-level properties. Circadian oscillation of mouse SCN organotypic slice cultures was monitored as PER2::LUC bioluminescence. SCN of three genetic backgrounds-wild-type, short-period CK1 $\varepsilon^{\text {Tau/Tau }}$ mutant, and long-period $F b x l 3^{A f h / A f h}$ mutant-all responded reversibly to pharmacological manipulation with period-altering compounds: picrotoxin, PF-670462 (4-[1-Cyclohexyl-4-(4-fluorophenyl)-1H-imidazol-5-yl]-2-pyrimidinamine dihydrochloride), and KNK437 (N-Formyl-3,4-methylenedioxybenzylidine-gamma-butyrolactam). This revealed a remarkably wide operating range of sustained periods extending across $25 \mathrm{~h}$, from $\leq 17 \mathrm{~h}$ to $>42 \mathrm{~h}$. Moreover, this range was maintained at network and single-cell levels. Development of a new technique for formal analysis of circadian waveform, first derivative analysis (FDA), revealed internal phase patterning to the circadian oscillation at these extreme periods and differential phase sensitivity of the SCN to genetic and pharmacological manipulations. For example, FDA of the CK1 $\varepsilon^{T a u / T a u}$ mutant SCN treated with the CK1 $\varepsilon$-specific inhibitor PF-4800567 (3-[(3-Chlorophenoxy)methyl]-1-(tetrahydro-2H-pyran-4-yl)-1H-pyrazolo[3,4-d]pyrimidin-4-amine hydrochloride) revealed that period acceleration in the mutant is due to inappropriately phased activity of the CK1 $\varepsilon$ isoform. In conclusion, extreme period manipulation reveals unprecedented elasticity and temporal structure of the SCN circadian oscillation.

Key words: bioluminescence; casein kinase; FBXL3; organotypic slice; period; picrotoxin

Significance Statement

The master circadian clock of the suprachiasmatic nucleus (SCN) encodes time-of-day information that allows mammals to predict and thereby adapt to daily environmental cycles. Using combined genetic and pharmacological interventions, we assessed the temporal elasticity of the SCN network. Despite having evolved to generate a $24 \mathrm{~h}$ circadian period, we show that the molecular clock is surprisingly elastic, able to reversibly sustain coherent periods between $\leq 17$ and $>42 \mathrm{~h}$ at the levels of individual cells and the overall circuit. Using quantitative techniques to analyze these extreme periodicities, we reveal that the oscillator progresses as a sequence of distinct stages. These findings reveal new properties of how the SCN functions as a network and should inform biological and mathematical analyses of circadian timekeeping.

\section{Introduction}

The paired suprachiasmatic nuclei (SCNs) are the principal circadian clock of mammals. Each consists of a small network of

This work was supported by UK Medical Research Council Grant MC_U105170643. We thank the biomedical staff at the Laboratory of Molecular Biology and Ares facilities for excellent technical support.
The authors declare no competing financial interests.

This article is freely available online through the $J$ Neurosci Author Open Choice option.

Correspondence should be addressed to Michael H. Hastings at the above address. E-mail: mha@mrc-Imb.cam.ac.uk.

D0I:10.1523/JNEUROSCI.0958-16.2016

Copyright $\odot 2016$ Patton et al.

This is an Open Access article distributed under the terms of the Creative Commons Attribution License Creative Commons Attribution 4.0 International, which permits unrestricted use, distribution and reproduction in any medium provided that the original work is properly attributed. 
$\sim 10,000$ neurons, and together they coordinate daily rhythms of activity and physiology that adapt individuals to the demands of the day/night cycle. In the absence of temporal cues, these rhythms free run with a circa (ca.) $24 \mathrm{~h}$ period, emphasizing the stability of the circadian system. The molecular clockwork of the SCN (and its subordinate clocks distributed across the body) consists of a transcriptional-translational feedback loop in which the Period (Per) and Cryptochrome (Cry) genes are negatively regulated by their protein products (Hastings et al., 2014). The period of the oscillation is determined, inter alia, by rates of expression and degradation of PER and CRY proteins (Maywood et al., 2011b), while ultimate coordination of behavior and physiology is mediated by the circadian cycle of spontaneous electrical firing by SCN neurons, regulated by the core molecular loop (Colwell, 2011).

The molecular oscillation can be monitored in real time in organotypic SCN slices using genetically encoded reporters, e.g., the PER2-luciferase fusion protein (PER2::LUC; Yoo et al., 2004). Remarkably, SCN tissue explants maintain free-running circadian rhythms more or less indefinitely in suitable culture conditions. This stability and robustness are conferred by tight network communication, mediated through electrical activity and neuropeptidergic signaling (Yamaguchi et al., 2003; Maywood et al., 2006, 2011a; Liu et al., 2007). Thus, synchronization of neuronal subpopulations across the nucleus maintains coherent ensemble molecular and electrophysiological rhythms.

Recently, the classic $\mathrm{GABA}_{\mathrm{A}}$-receptor antagonist picrotoxin was shown to accelerate the SCN clock, independently of its canonical role in antagonizing $\mathrm{GABA}_{\mathrm{A}}$ receptors (Freeman et al., 2013). In contrast to pharmacological acceleration, inhibition of casein kinase $1 \varepsilon / \delta(\mathrm{CK} 1 \varepsilon / \delta)$ by the antagonist PF-670462 (4-[1-Cyclohexyl-4-(4-fluorophenyl)-1 H-imidazol-5-yl]2-pyrimidinamine dihydrochloride) lengthens the period of explant SCN slices (Meng et al., 2010), as does KNK437 (NFormyl-3,4-methylenedioxy-benzylidine-gamma-butyrolactam), an inhibitor of heat-shock factor 1 (HSF1; Buhr et al., 2010). Several genetic mutations also alter the period of the SCN oscillation. The Tau mutation, originally identified in Syrian hamsters, causes the SCN clock to run at $20 \mathrm{~h}$ (Ralph and Menaker, 1988) due to a point mutation within the CK1 $\varepsilon$ gene (Lowrey et al., 2000). The CK1 $\varepsilon^{\text {Tau/Tau }}$ mutation has been genetically engineered in mice, and accelerates the clock through accelerated degradation of PER protein, thereby truncating the phase of transcriptional repression (Meng et al., 2008). In contrast, the Afterhours (Afh) mutation in the F-box/LRR-repeat protein $3\left(\mathrm{Fbxl}^{\mathrm{Afh} / \mathrm{Afh}}\right)$ slows CRY degradation and prolongs the transcriptional repression phase, driving the SCN to a ca. $28 \mathrm{~h}$ period (Godinho et al., 2007). Thus, $\mathrm{Fb} \times 13^{A f h / A f h}$ and $C K 1 \varepsilon^{\text {Tau/Tau }}$ have opposing actions, acting independently and additively (Maywood et al., 2011b).

The aim of this study was to combine these pharmacological and genetic manipulations to push the SCN clockwork beyond its normal period of oscillation, and by doing so reveal unanticipated cell-autonomous and emergent, circuit-level properties. To facilitate this, we developed a novel approach to analyze the profile of the PER2::LUC bioluminescent waveform, employing first derivative analysis (FDA). By conducting such a "stress test," we anticipated three possible outcomes: complete arrhythmia; asynchrony of competent cellular oscillators in an incompetent circuit; or maintained cell-autonomous and circuit-level rhythms of extreme period, should the network contain sufficient temporal elasticity. We report that both the cell-autonomous and the circuit-level functions of the SCN are extremely robust in the face of these extreme perturbations, with an operating range of $>25 \mathrm{~h}$, extending from $<17 \mathrm{~h}$ to $>42 \mathrm{~h}$. FDA waveform analysis at extreme periods revealed internal phase patterning to the circadian oscillation and differential phase sensitivity of the SCN to genetic and pharmacological manipulations. This was validated by examination of the interplay between the CK1 $1 \varepsilon^{\text {Tau/Tau }}$ mutation and the specific CK1 $\varepsilon$ inhibitor PF-4800567. Thus, the SCN clock functions as a sequence of distinct stages and is remarkably robust and elastic at both cell-autonomous and circuit levels.

\section{Materials and Methods}

Slice preparation. All animal work was licensed under the UK 1986 Animals (Scientific Procedures) Act and subject to local ethical review. SCN slices were isolated from $300-\mu \mathrm{m}$-thick coronal hypothalamic slices prepared from postnatal day 8 (P8) to P10 mice (either sex) and cultured on Millicell tissue culture inserts (PICMORG50, Millipore) for at least a week at $37^{\circ} \mathrm{C}$, as previously described (Yamazaki et al., 2002; Hastings et al., 2005; Brancaccio et al., 2013). Slices were prepared from wild-type and circadian mutant mice, $C K 1 \varepsilon^{\text {Tau/Tau }}$ and Fblx $3^{A f h / A f h}$, carrying the PER2::LUC reporter.

Bioluminescent recordings. Slices were transferred to individual dishes containing DMEM (Sigma-Aldrich) supplemented with Glutamax, penicillin/streptomycin, FCS, B27, and beetle luciferin as described previously (Hastings et al., 2005; Brancaccio et al., 2013). Dishes were sealed with a glass coverslip and vacuum grease, placed under a photomultiplier tube (PMT; H9319-11 photon-counting head, Hamamatsu), and maintained at $37^{\circ} \mathrm{C}$ in a nonhumidified incubator. Bioluminescence recordings were made at $1 \mathrm{~s}$ intervals, binned into $6 \mathrm{~min}$ epochs for subsequent analysis and monitored in real-time for at least five full cycles at each condition (baseline, pharmacological manipulation, and washout). Immortalized fibroblasts produced from lungs of PER2::LUC mice (kindly provided by J. O'Neill, MRC Laboratory of Molecular Biology) were seeded into $33 \mathrm{~mm}$ dishes to confluence (O'Neill and Hastings, 2008) $24 \mathrm{~h}$ before entrainment. Medium was then changed to supplemented DMEM (as detailed for SCN explants), and dishes were sealed before transfer to a computer-controlled incubator (Galaxy 48R, New Brunswick Scientific) for entrainment to a temperature cycle $\left(12 \mathrm{~h} 32^{\circ} \mathrm{C}, 12 \mathrm{~h}\right.$ $37^{\circ} \mathrm{C}$ ) over four cycles. On the final warm phase, cells were treated with either $500 \mu \mathrm{M}$ picrotoxin or $0.5 \%$ DMSO and immediately transferred to PMTs to free run at $37^{\circ} \mathrm{C}$, and continuous recordings of bioluminescence were made over $5 \mathrm{~d}$.

For single SCN cell analysis, sealed dishes were transferred to the heated stage of an upright microscope, and bioluminescence was visualized by CCD camera. Time-lapse images of bioluminescent signals were taken at $1 \mathrm{~h}$ intervals over five cycles per condition. When $1 \mu \mathrm{M}$ tetrodotoxin (TTX) was applied to slices in the presence of test compounds, an additional $0.1 \mu \mathrm{M}$ luciferin was added to the medium to attenuate the damping of the molecular oscillation that is characteristic of TTX treatment (Yamaguchi et al., 2003). After the experiment, individual ROI analysis was performed using the Semi-Automated Routines for Functional Image Analysis (SARFIA; Dorostkar et al., 2010) package in IGOR Pro as described previously (Brancaccio et al., 2013), and for each slice, $>100$ ROIs were identified. Center-of-luminescence (CoL) analysis was performed in IGOR Pro using custom in-house scripts to detect the frame-by-frame $X Y$ coordinates of the $\mathrm{CoL}$ as described previously (Brancaccio et al., 2013). Path indexes of the trajectories were calculated as total pixel excursion divided by the period of the oscillation, before normalization to the relative bioluminescent pixel area of the nucleus measured and expressed as a fraction of the baseline to account for differences in relative magnification between microscopes. All image analysis was performed in FIJI (Schindelin et al., 2012) and IGOR Pro (WaveMetrics).

Drug treatments. At least five full cycles after the start of an experiment, all applications were made as a 1:1000 to 5:1000 dilution of the compound plus vehicle directly into the baseline medium. Drugs and corresponding vehicle treatments were run simultaneously for each genotype. Picrotoxin and DMSO (final concentration, $\leq 0.5 \%$ ) were purchased from Sigma-Aldrich; PF-670462 and PF-4800567 (3-[(3- 
Chlorophenoxy)methyl]-1-(tetrahydro-2H-pyran-4-yl)- $1 H$-pyrazolo [3,4-d]pyrimidin-4-amine hydrochloride) from were purchased from R\&D Systems; and KNK437, gabazine (SR-95531), and TTX citrate from were purchased from Cambridge Bioscience. Period-altering drugs were applied at maximally effective concentrations of $100 \mu \mathrm{M}$ for picrotoxin, 1 $\mu \mathrm{M}$ for PF-670462, and $100 \mu \mathrm{M}$ for KNK437.

Period analysis. Period was analyzed using the Biological Rhythms Analysis Software System software running on the BioDARE platform at the University of Edinburgh (courtesy of A. Millar; http://www.biodare. ed.ac.uk/; Moore et al., 2014; Zielinski et al., 2014). The first $12 \mathrm{~h}$ of all recordings were ignored to remove any artifacts arising from medium change, the incubator being opened, etc. PMT data were analyzed as raw data without any detrending except in the case of fibroblast data, which were detrended with a $12 \mathrm{~h}$ moving average and smoothed with a centered moving average. The first $24 \mathrm{~h}$ of fibroblast data following entrainment were ignored, so that only free-running rhythms were analyzed. Relative amplitude error (RAE) and phase were obtained directly from the BioDARE output. All single-cell CCD data were detrended by a $12 \mathrm{~h}$ moving average and smoothed by a centered moving average before analysis in BioDARE to resolve low amplitude rhythms within the longterm recording. Where required, phase coherence was checked by manual calculation and resulted in comparable mean vector lengths to Rayleigh analysis performed using BioDARE-calculated values. Relative period range widths for single-cell experiments were calculated by subtracting the minimum period from the maximum period from the BioDARE-calculated values and dividing the resulting range width by the population mean period for that $\mathrm{SCN}$.

Waveform analysis. Waveform analysis was performed on individual peaks, with period and peak amplitude normalized to allow direct comparison across conditions. Peak amplitude was normalized between the 0 and 1 using unity-based normalization. The first derivative was calculated directly from the data using the Differentiate function in IGOR Pro. Data were integrated into bins of normalized period with 0.02 period bin widths to directly make comparisons across genotype and pharmacological conditions. FDA baseline subtraction (FDA-S) analysis was applied by subtracting the binned baseline values from the binned treatment values to map changes at each time point arising from vehicle or drug treatment. Peak identification was performed using GraphPad Prism to return peak coordinates from baseline-subtracted curves.

Statistical analysis. Two-way ANOVA with Šidák's multiple comparisons test was performed on all FDA data sets to determine statistical significance within and between conditions. Where appropriate, repeated measures one-way ANOVA with the Greenhouse-Greisser correction or paired two-tailed $t$ tests were applied to in-slice comparisons. One-way ANOVA and unpaired two-tailed $t$ tests were applied to comparisons between different treatment populations. Where multiple comparisons were made with ANOVA, $p$ values were adjusted for multiple comparisons using the Holm-S̆idák multiple comparison test. Rayleigh analyses were applied by converting phase data to circular data within Microsoft Excel, and performed on the native time base for the data sets, as determined by period analysis. Circular Rayleigh plots were produced using Oriana software (Kovach Computing Services). All statistics and data analyses were performed in GraphPad Prism, WaveMetrics IGOR Pro, and Microsoft Excel for Mac 2008.

\section{Results}

The SCN molecular clockwork can sustain extreme periods under combined genetic and pharmacological manipulations The first aim of mapping the limits of SCN circadian period was to explore the operating range and robustness of the clock mechanism. Second, it was anticipated that pushing the SCN to extreme periods could selectively reveal critical phases in the oscillation, thereby identifying hidden aspects of its underlying structure. Using three compounds known to be efficacious in altering the period of the SCN: picrotoxin (Freeman et al., 2013); PF-670462, an inhibitor of CK1 $1 \varepsilon / \delta$ (Meng et al., 2010); and KNK437, an inhibitor of HSF1 (Buhr et al., 2010), it was important to demonstrate the reversibility of any responses, to confirm that even at extreme periods the intrinsic mechanisms of the SCN remained fully competent and resilient to return to baseline. None of the compounds had a permanent effect on the period of PER2::LUC bioluminescence rhythms of wild-type SCNs (grouped baseline, $24.63 \pm 0.10 \mathrm{~h}$ vs grouped washout, $24.69 \pm$ $0.08 \mathrm{~h} ; p=0.65)$. Picrotoxin $(100 \mu \mathrm{M})$ reversibly shortened the period of individual wild-type SCNs (pretreatment vs treatment, $25.07 \pm 0.22 \mathrm{~h}$ vs $20.36 \pm 0.34 \mathrm{~h} ; p<0.01 ; n=8$ ), whereas PF-670462 $(1 \mu \mathrm{M})$ and KNK437 $(100 \mu \mathrm{M})$ reversibly lengthened period (PF-670462, $24.51 \pm 0.15 \mathrm{~h}$ vs $30.94 \pm 0.26 \mathrm{~h}, p<0.01$, $n=8$; KNK437, $24.45 \pm 0.08 \mathrm{~h}$ vs $33.06 \pm 0.21 \mathrm{~h}, p<0.01, n=$ 8; Fig. 1A). The respective period changes were significantly different from the minimal effects of vehicle delivery $(100 \mu \mathrm{M}$ picrotoxin vs $0.1 \%$ DMSO, $p<0.01, n=8 / 8 ; 1 \mu \mathrm{M}$ PF-670462 vs $0.01 \% \mathrm{H}_{2} \mathrm{O}, p<0.01, n=8 / 8 ; 100 \mu \mathrm{M}$ KNK437 vs $0.5 \%$ DMSO, $p<0.01, n=8 / 8$ ).

Pharmacological manipulation was then applied to genetically modified SCNs, which exhibited the expected short $\left(C K 1 \varepsilon^{\text {Tau/Tau }}, 20.4 \pm 0.28 \mathrm{~h}\right)$ and long $\left(F b x l 3^{\text {Afh/Afh }}, 28.67 \pm\right.$ $0.20 \mathrm{~h})$ periods relative to wild-type slices $(24.63 \pm 0.10 \mathrm{~h}$; Fig. $1 B, C$, vehicle traces). Picrotoxin $(100 \mu \mathrm{M})$ shortened the period by ca. $4 \mathrm{~h}$ in $C K 1 \varepsilon^{\mathrm{Tau} / \mathrm{Tau}}$ SCNs, leading to an unprecedentedly short composite period of ca. $17 \mathrm{~h}$ (Fig. 1D; baseline, $20.43 \pm$ $0.28 \mathrm{~h}$ vs treatment, $17.17 \pm 0.31 \mathrm{~h} ; p<0.01 ; n=8$ ). The effect was fully reversed on washout, confirming that even at this extreme acceleration, the SCN maintained elasticity (pretreatment baseline, $20.22 \pm 0.74 \mathrm{~h}$ vs washout, $20.48 \pm 0.64 \mathrm{~h} ; p=0.81 ; n=$ 8). Picrotoxin accelerated the long-period mutant $F b x / 3^{A f h / A f h}$ by $\sim 8.0 \mathrm{~h}$ (Fig. $1 C, D$; baseline, $28.67 \pm 0.20 \mathrm{~h}$ vs treatment, $20.67 \pm$ $0.21 \mathrm{~h} ; p<0.01 ; n=8)$. Surprisingly, this was comparable to the period of wild-type SCNs treated with picrotoxin; i.e., the effect of the mutation was completely masked (Fig. $1 A, C, D$; wild-type vs Fbxl3 $\left.3^{A f h / A f h}, p=0.46, n=8 / 8\right)$. Comparison of the effects of picrotoxin across genotypes [Fbxl3 $]^{A f h / A f h}, 8.00$ in $28.67 \mathrm{~h}(28 \%)$; wild-type, 4.71 in $25.07 \mathrm{~h}$ (19\%); CK1 $\varepsilon^{\text {Tau/Tau }}, 3.26$ in $20.43 \mathrm{~h}$ $(16 \%)$ ] suggests that pharmacologically induced period shortening by picrotoxin was manifest neither as a simple proportional scaling across the cycle nor as a discrete reduction in solar hours (Fig. 1E).

To probe the upper limit of SCN resilience, the two periodlengthening compounds, PF-670462 and KNK437, were applied, and both slowed the circadian oscillation of SCN explants from all three genotypes (Fig. 1A-D). Application of $100 \mu \mathrm{M}$ KNK437 caused the greatest deceleration of the clock, slowing the $\mathrm{Fb} \times 13^{\text {Afh/Afh }}$ oscillation by around $14 \mathrm{~h}$ to create SCNs with an unprecedentedly slow period of ca. $42 \mathrm{~h}$ (Fig. $1 C, D$; baseline, $28.53 \pm 0.14 \mathrm{~h}$ vs treatment, $42.38 \pm 0.93 \mathrm{~h} ; p<0.01 ; n=8$ ). Again, in comparing across genotypes the period lengthening by either PF-670462 [Fbxl $3^{A f h / A f h}, 9.36$ in 28.6 h (33\%); wild-type, 6.43 in $24.51 \mathrm{~h}(26 \%) ; C K 1 \varepsilon^{\text {Tau/Tau }}, 7.40$ in $\left.20.42 \mathrm{~h}(36 \%)\right]$ or KNK437 [Fbxl3 ${ }^{A f h / A f h}, 13.85$ in $28.53 \mathrm{~h}(49 \%)$; wild-type, 8.61 in $24.45 \mathrm{~h}(35 \%)$; CK1 $\varepsilon^{\text {Tau/Tau }}, 9.55$ in $20.24 \mathrm{~h}(47 \%)$ ], it was clear that these changes were manifested neither by consistent proportional nor discrete absolute increases in period (Fig. 1E). Thus, across the three genotypes, each drug changed period in a qualitatively similar but unequal manner: the response to pharmacological treatments relied on the condition of the oscillator; i.e., period changes were manifested as an interaction between genetics and pharmacology.

Interestingly, this genetic and pharmacological interaction was most obvious with the period-lengthening compounds where both KNK437 and PF-670462 had larger effects in mutant than in wild-type SCN (Fig. 1E; wild type vs CK1 $\varepsilon^{\text {Tau/Tau }}, 1 \mu \mathrm{M}$ 
A

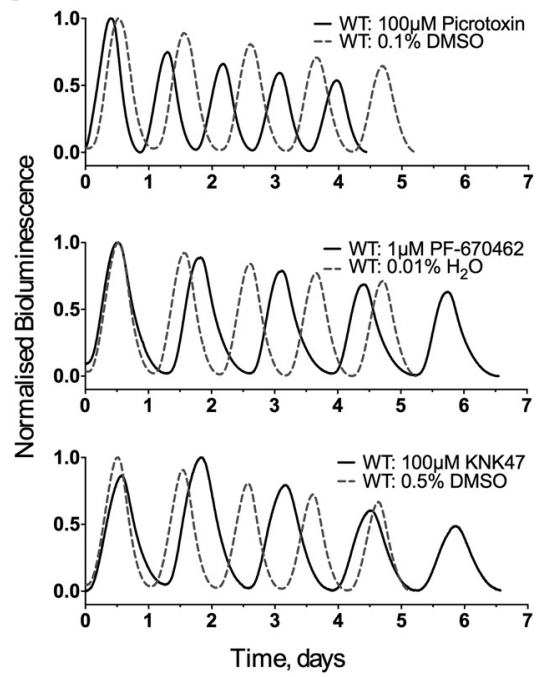

D

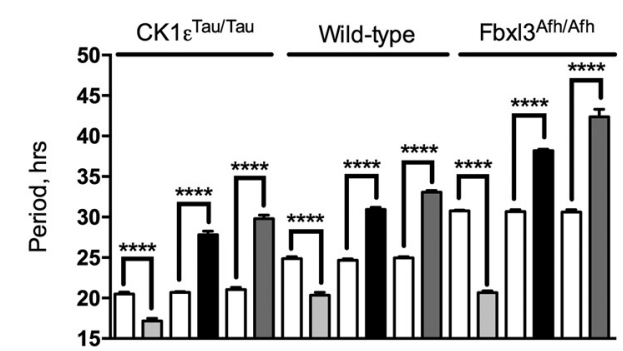

Vehicle + $++_{-}++_{-}++_{-}++_{-}+-$

Picrotoxin - + - - - + - - - + + - -

PF-670462 _ - + - - - + + - - - + -

KNK437 _ _ _ $+\ldots+\ldots+\ldots$

G

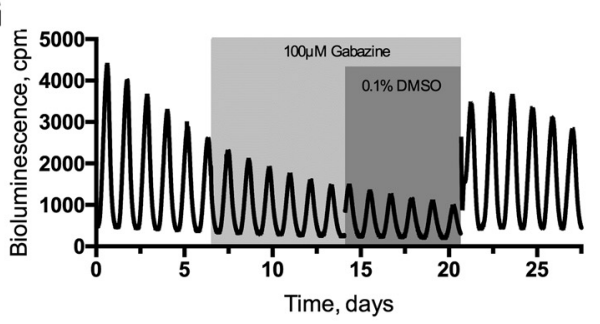

I

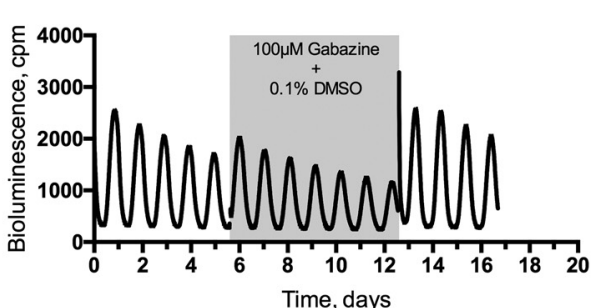

$\mathbf{K}$

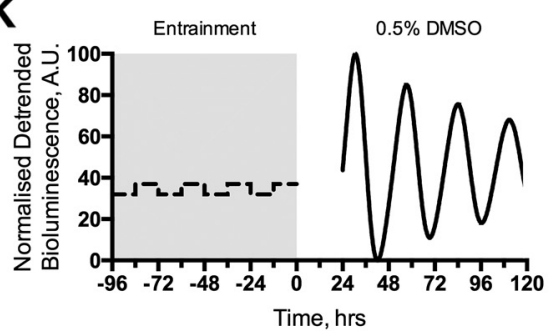

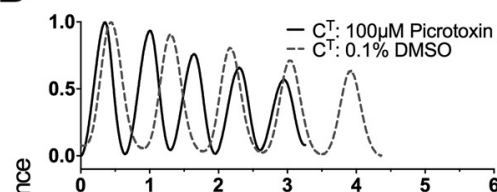

C
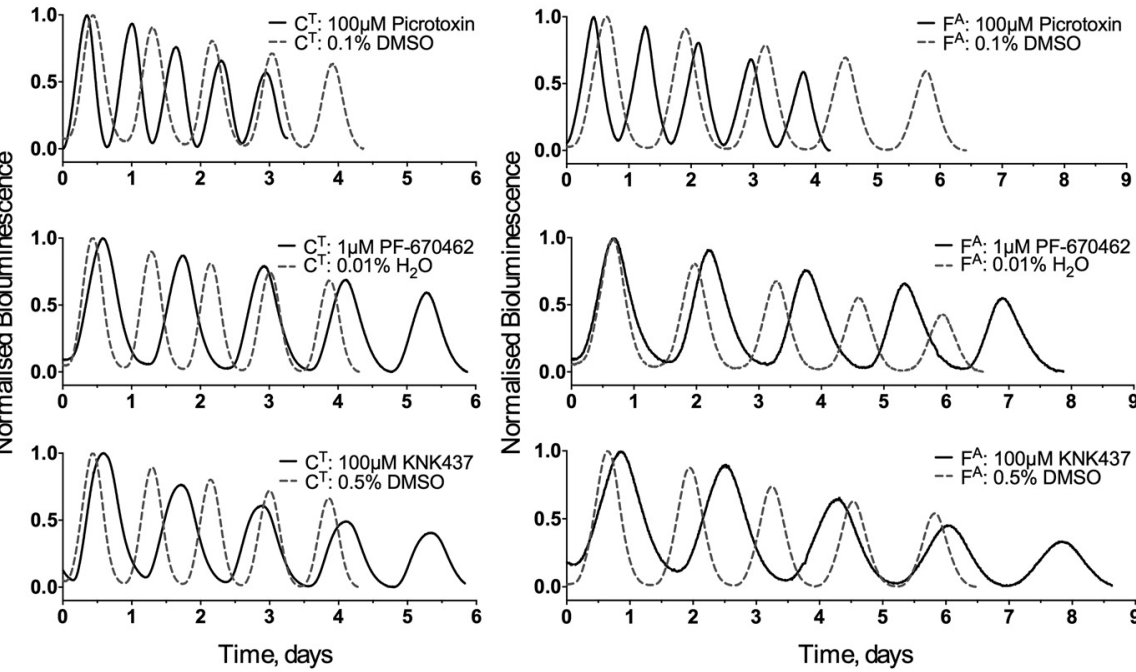

E

$\mathbf{F}$
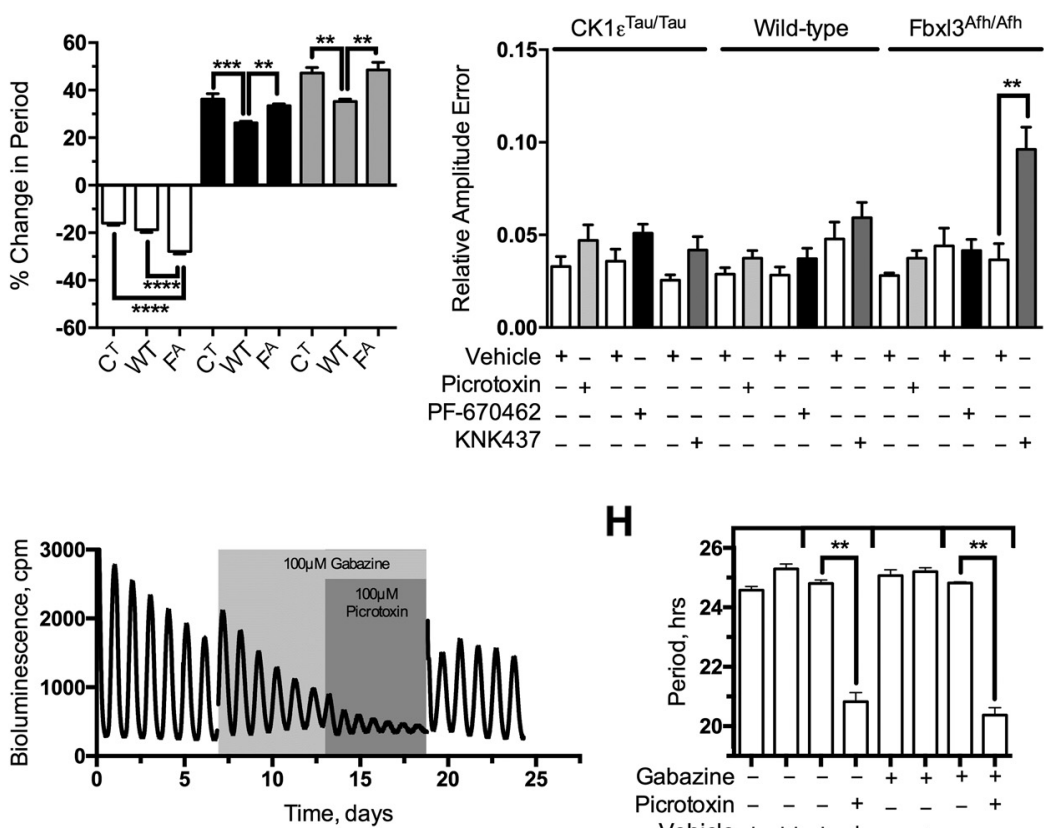

H
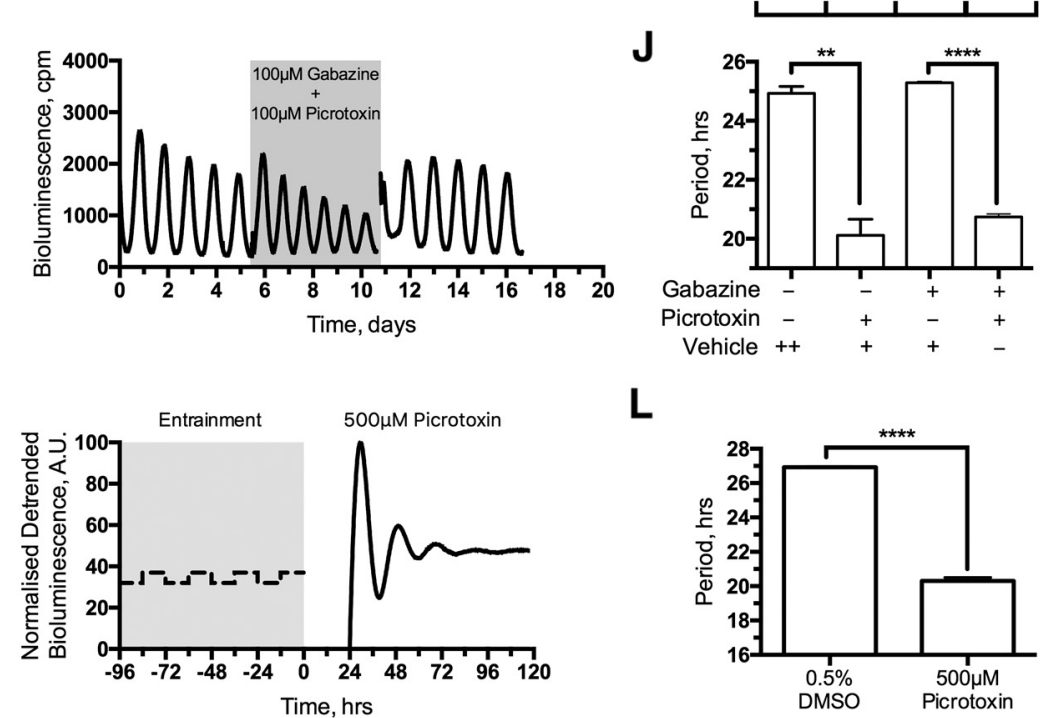

$\mathbf{L}$
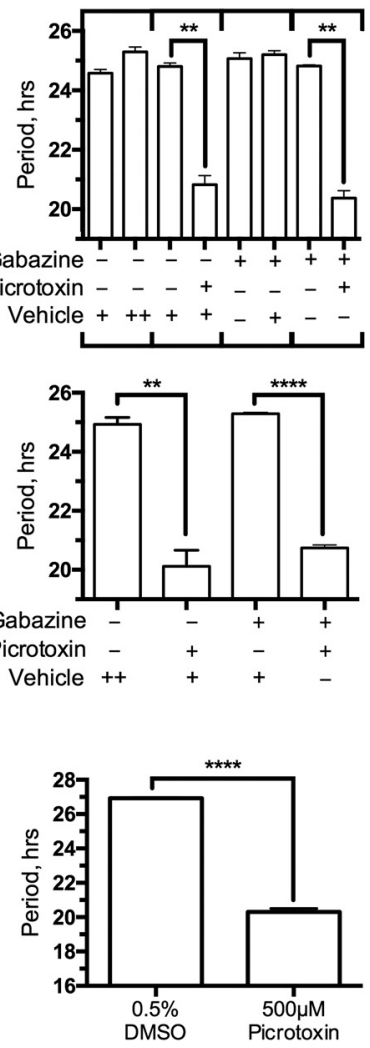
PF670462, $p<0.01, n=8 / 8 ; 100 \mu \mathrm{M} \mathrm{KNK437,} p<0.01, n=8 / 8$; wild type vs Fbxl3 $3^{\text {Afh/Afh }}, 1 \mu \mathrm{M}$ PF670462, $p<0.01, n=8 / 8 ; 100$ $\mu \mathrm{M}$ KNK437, $p<0.01, n=8 / 8$ ). This suggests that these compounds have an upper limit of about 35 or $48 \%$ (for PF-670462 and KNK437, respectively) in proportionally extending the oscillation (Fig. 1E; CK1 $\varepsilon^{\text {Tau/Tau }}$ vs Fbxl3 ${ }^{\text {Afh/Afh }}, 1 \mu \mathrm{M}$ PF-670462, $p=$ $0.18, n=8 / 8 ; 100 \mu \mathrm{M} \mathrm{KNK437,} p=0.70, n=8 / 8$ ). As seen for period shortening, with period lengthening there was also a significant interaction between pharmacology and genetic background that is not simply a proportional scaling. This is not wholly unexpected for the interaction between PF-670462 and the CK1 $\varepsilon^{\text {Tau/Tau }}$ conditions where PF-670462 acts on the CK1mediated axis of circadian timekeeping (Meng et al., 2010). It is, however, surprising that there is a larger proportional effect in both $C K 1 \varepsilon^{\text {Tau/Tau }}$ and $F b x l 3^{\text {Afh/Afh }}$ slices treated with KNK437, where a relationship between genetics and pharmacology would not necessarily be expected.

Thus, molecular timekeeping in the SCN, a biological clock that has evolved to operate at a period of ca. $24 \mathrm{~h}$, has limits of operation that span at least between 17 and $42 \mathrm{~h}$ (a range of $25 \mathrm{~h}$, i.e., $>100 \%$ of the normal period), and even when pushed to such extremes, it retains complete elasticity. Moreover, visual inspection of the bioluminescence curves suggested that at these extreme periods, the oscillation maintained coherence. This was confirmed by the RAE, an inverse index of overall coherence (Fig. $1 F)$. In all cases except one, RAE was unaffected by drug application when compared to baseline (data not shown) or to vehicle treatment (Fig. $1 F$ ). The exception was seen in Fbxl3 ${ }^{\text {Afh } / A f h}$ SCNs treated with $100 \mu \mathrm{M} \mathrm{KNK437} \mathrm{(baseline} \mathrm{RAE,} 0.044 \pm 0.005$ vs treatment RAE, $0.096 \pm 0.012 ; p=0.011 ; n=8$; Fig. $1 F)$. This reduced coherence at the extremely long period suggests that the upper limit of the temporal elasticity of the SCN network was near. It should be noted, however, that in genetically disrupted SCNs, for example, with combined null mutations of Per1 and Per2, or deletion of VIP or Vipr2 (which encodes the receptor for

$\leftarrow$

Figure 1. Genetic and pharmacological manipulation of the SCN period greatly extends the operational range of explant SCN slices. A-C, Example PMT traces showing normalized bioluminescence for treatment intervals. Treatments are as follows: $100 \mu \mathrm{m}$ picrotoxin/0.1\% DMSO (top), $1 \mu \mathrm{m} \mathrm{PF}-670462 / 0.01 \% \mathrm{H}_{2} \mathrm{O}$ (middle), and $100 \mu \mathrm{m} \mathrm{KNK437/0.5 \%} \mathrm{DMSO} \mathrm{(bottom).} \mathrm{Treat-}$ ment (solid black) is overlaid with vehicle traces (dashed gray) grouped by genotype. $A$, Wildtype PER2::LUC (WT). B, CK1 $\varepsilon^{\text {Tau/Tau }} \times$ PER2:::LUC ( $\left(C^{\top}\right)$. C, FbxI3 $3^{\text {Afh/Afh }} \times$ PER2:::LUC $\left(F^{A}\right)$. $D$, Summary period data expressed as mean \pm SEM from each treatment condition grouped by genotype. Treatments accompanied by their specific vehicles (white) are $100 \mu \mathrm{m}$ picrotoxin (light gray), $1 \mu \mathrm{M}$ PF-670462 (black), and $100 \mu \mathrm{M}$ KNK437 (dark gray), as indicated. E, Summary data expressing the proportional change in period expressed the percentage change from baseline period induced by period-altering compounds and expressed as mean \pm SEM. Bars are grouped by pharmacological treatment: $100 \mu \mathrm{m}$ picrotoxin (white), $1 \mu \mathrm{m}$ PF-670462 (black), and $100 \mu \mathrm{m}$ KNK437 (gray). Genotypes are identified as CK1 $\varepsilon^{\text {Tau/Tau }} \times$ PER2:::LUC, wild-type PER2:::LUC (WT) and Fbx/3 $3^{\text {ffh/Afh }} \times$ PER2:::LUC. F, Summary RAE expressed as mean \pm SEM from each condition grouped by genotype. Treatments accompanied by their specific vehicles (white) are $100 \mu \mathrm{m}$ picrotoxin (light gray), $1 \mu \mathrm{m}$ PF-670462 (black), and $100 \mu \mathrm{m}$ KNK437 (dark gray), as indicated. G, Example PMT traces for continuous wild-type SCN explant experiments cotreated in series with $100 \mu \mathrm{m}$ gabazine and $100 \mu \mathrm{m}$ picrotoxin (right) and $100 \mu \mathrm{m}$ gabazine and $0.1 \%$ DMSO (left). Treatment intervals are indicated by gray shaded regions. $\boldsymbol{H}$, Summary period data as mean \pm SEM for series cotreatment experiment. Treatments are as indicated, and in-series treatments are grouped by brackets. I, Example PMT traces for continuous wildtype SCN explant experiments cotreated with $100 \mu \mathrm{m}$ gabazine and $100 \mu \mathrm{m}$ picrotoxin (right) and $100 \mu \mathrm{m}$ gabazine and $0.1 \%$ DMSO (left). Treatment intervals are indicated by gray shaded regions. J, Summary period data as mean \pm SEM for cotreated experiments. Treatments are as indicated. $\boldsymbol{K}$, Fibroblast representative traces (detrended) for $500 \mu \mathrm{m}$ picrotoxin treatment (right) and $0.5 \%$ DMSO treatment (left). $L$, Summary period data for fibroblast experiments as indicated. $n$ values are detailed throughout the text. ${ }^{*} p<0.05,{ }^{* *} p<0.01,{ }^{* * *} p<0.001$, ${ }^{* * * *} p<0.0001$.
VIP), the RAE lies in the range of 0.15 to 0.20 (Maywood et al., 2011a, 2014), far beyond that seen here. Thus, the circuit-level circadian functions of the SCN are highly elastic and can sustain molecular oscillations that range in period between 17 and $42 \mathrm{~h}$ without a significant loss of temporal coherence.

The SCN network is predominately a GABA-ergic circuit, and picrotoxin is a classical $\mathrm{GABA}_{\mathrm{A}}$-receptor antagonist. Although the role of $\mathrm{GABA}_{\mathrm{A}}$-receptor antagonism in reducing period has been discounted previously (Freeman et al., 2013), the precise role of GABA in SCN timekeeping is still unknown. To further determine that period effects are due to an as yet unknown target of picrotoxin, wild-type slices were cotreated with the $\mathrm{GABA}_{\mathrm{A}}$-receptor antagonist gabazine (SR-95531) and picrotoxin in two configurations: serial and simultaneous treatments. With serial treatment (Fig. $1 G, H$ ), slices received $100 \mu \mathrm{M}$ gabazine for five cycles before $100 \mu \mathrm{M}$ picrotoxin or $0.1 \%$ DMSO was applied (Fig. 1G). Preantagonism of $\mathrm{GABA}_{\mathrm{A}}$ receptors did not induce a period change (baseline, $24.60 \pm 0.08 \mathrm{~h}$ vs $100 \mu \mathrm{M}$ gabazine, $24.82 \pm 0.04 ; p=0.18 ; n=3)$, and furthermore, this did not occlude (100 $\mu \mathrm{M}$ gabazine alone vs with $100 \mu \mathrm{M}$ picrotoxin, $p<$ $0.01, n=3)$ or attenuate the action of picrotoxin $(100 \mu \mathrm{M}$ picrotoxin, $100 \mu \mathrm{M}$ gabazine pretreatment vs $0.1 \%$ DMSO pretreatment; $p=0.32 ; n=3 / 3$; Fig. $1 H$ ). In the simultaneous treatment configuration (to control for any potential loss of efficacy in gabazine in the serial treatment configuration), gabazine was coapplied to slices with either picrotoxin or DMSO (Fig. 1I). Again, gabazine did not occlude (baseline, $24.50 \pm 0.07 \mathrm{~h}$ vs $100 \mu \mathrm{M}$ gabazine/100 $\mu \mathrm{M}$ picrotoxin, $20.74 \pm 0.10 \mathrm{~h} ; p<0.01 ; n=3 ; 100$ $\mu \mathrm{M}$ gabazine coapplied, $100 \mu \mathrm{M}$ picrotoxin vs $0.1 \%$ DMSO, $p<$ $0.01, n=3 / 3$ ) or attenuate the picrotoxin-induced period change (100 $\mu \mathrm{M}$ picrotoxin coapplied, $100 \mu \mathrm{M}$ gabazine vs $0.1 \%$ DMSO, $p=0.33, n=3 / 3$; Fig. $1 J)$. Thus, acceleration by picrotoxin in the $\mathrm{SCN}$ is not induced via $\mathrm{GABA}_{\mathrm{A}}$-receptor antagonism. To further confirm that picrotoxin affects the circadian clock directly and does not act via GABA-ergic synaptic signaling, PER2::LUC fibroblasts were treated with either $500 \mu \mathrm{M}$ picrotoxin or $0.5 \%$ DMSO immediately following four cycles of temperature entrainment (Fig. $1 \mathrm{~K})$. Consistent with previous reports (Freeman et al., 2013), $500 \mu \mathrm{M}$ picrotoxin caused a reduction of period compared to vehicle treatment $(500 \mu \mathrm{M}$ picrotoxin vs $0.5 \%$ DMSO, $p<0.01, n=6 / 6$; Fig. $1 L$ ). These experiments confirm that antagonism of synaptic $\mathrm{GABA}_{\mathrm{A}}$ receptors by picrotoxin is not a requisite for setting period length.

\section{Manipulating the period alters the waveform of the circadian PER2 bioluminescence profile}

Having created SCNs with extreme circadian periods, the next aim was to use them to probe the internal structure of the oscillation. Theoretically, changing the period could be achieved in one of two ways: first, by a global proportional scaling of the oscillation across all phases, and second, by a phase-specific scaling where different phases are more sensitive to particular interventions. The former would be compatible with traditional parametric models of the oscillator (Fuhr et al., 2015), whereas the latter would indicate it to be a series of distinct stages, and indeed the interaction between drug and genotype in setting SCN circadian period is supportive of the latter. To discriminate directly and quantitatively between these alternatives in genetically modified SCNs, individual cycles were peak aligned with the wild-type condition (peak PER2::LUC is circadian time 12, i.e., subjective dusk; Fig. $2 A-C$, top). When plotted in solar time, this revealed that $C K 1 \varepsilon^{\text {Tau/Tau }}$ and $F b x l 3^{A f h / A f h}$ produced phasespecific effects relative to peak bioluminescent activity (Fig. 
A
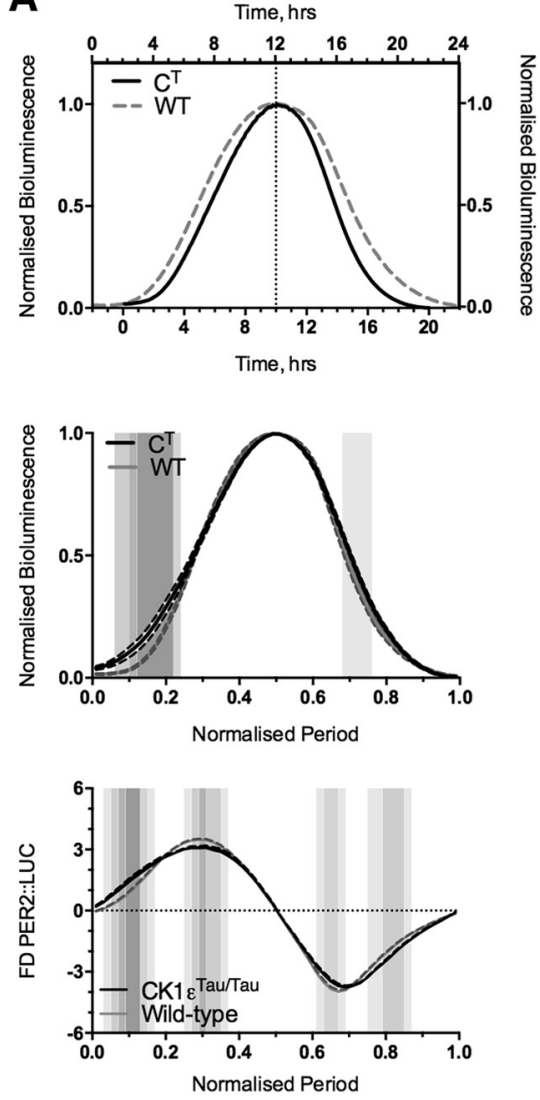

D

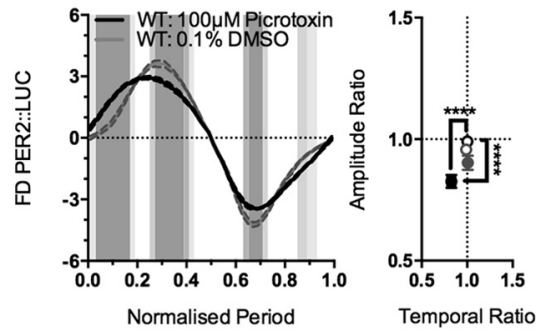

G

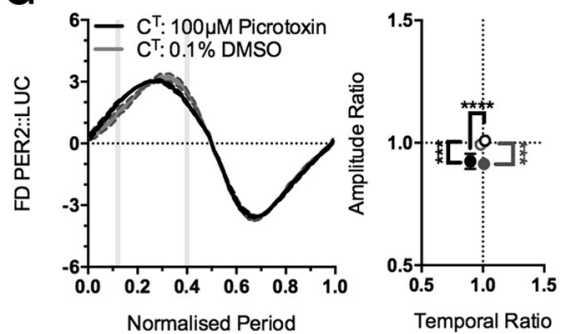

J

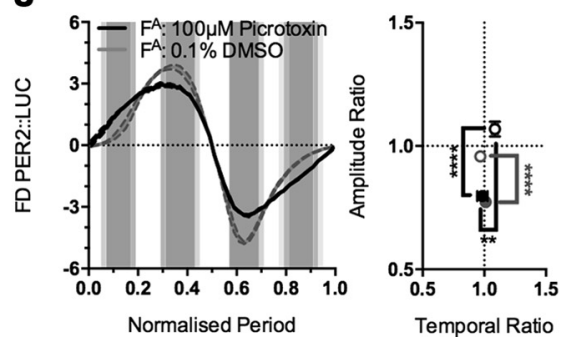

B
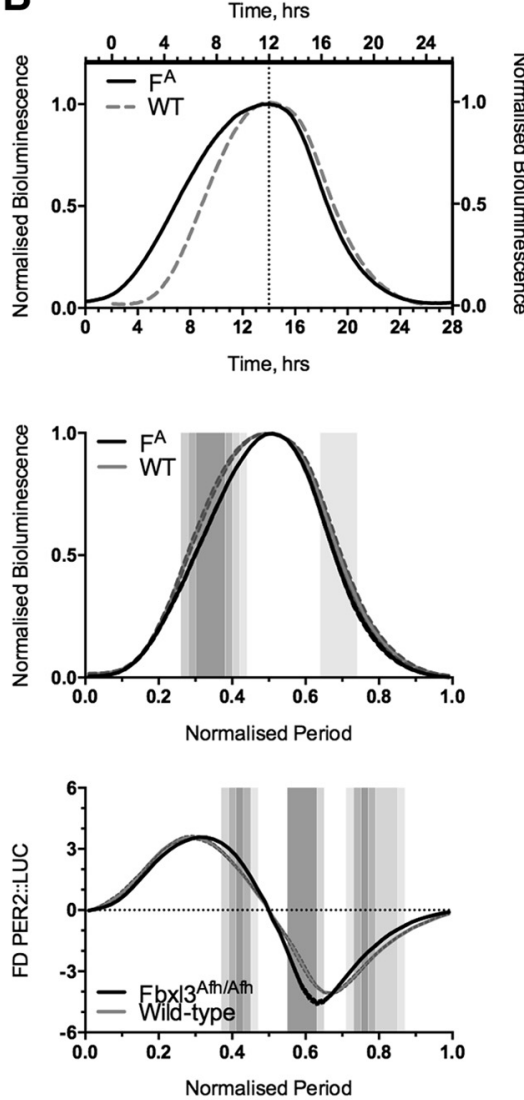

E

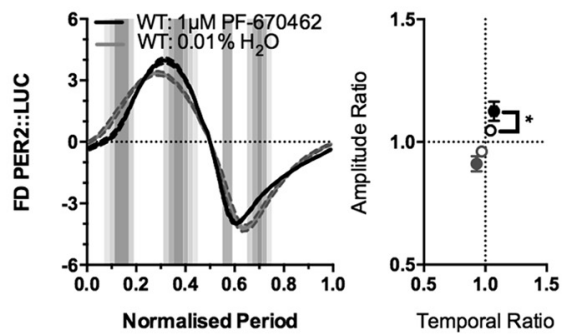

H
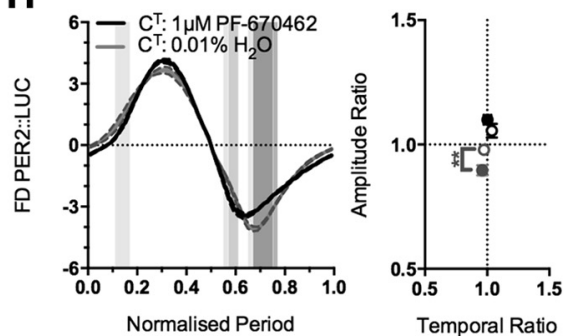

K

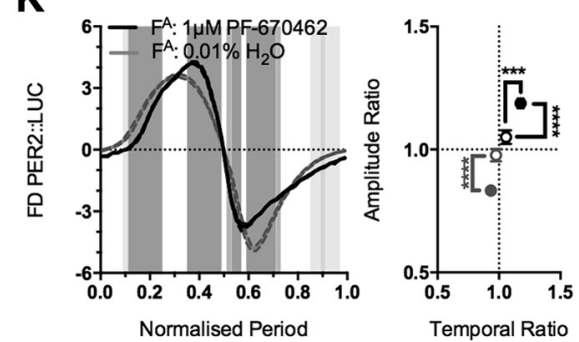

C
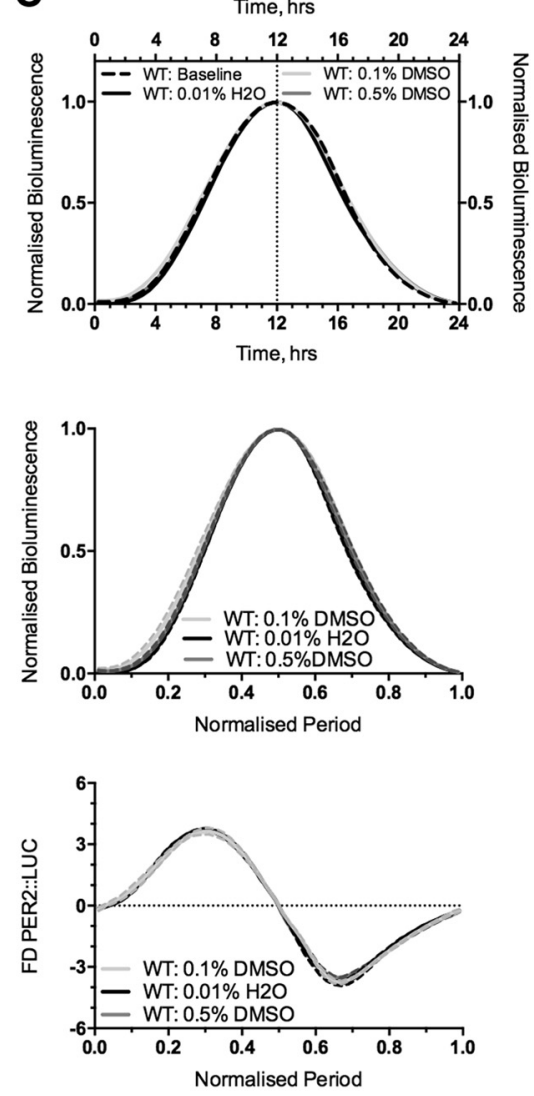

F

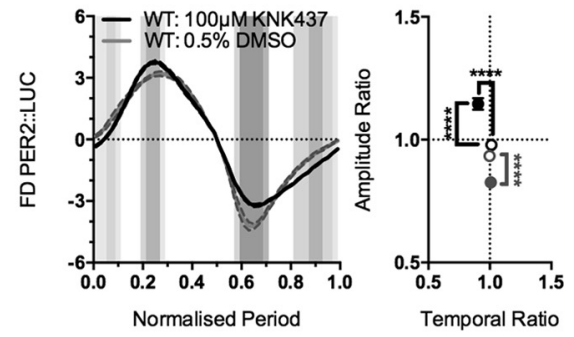

I

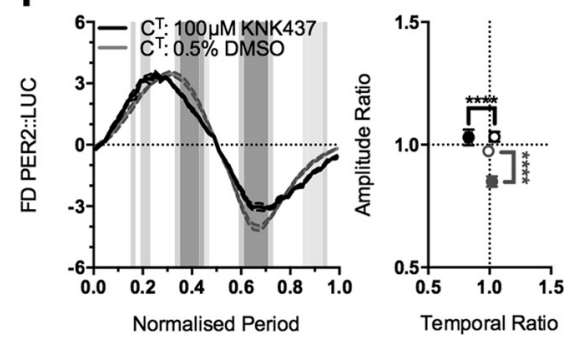

L

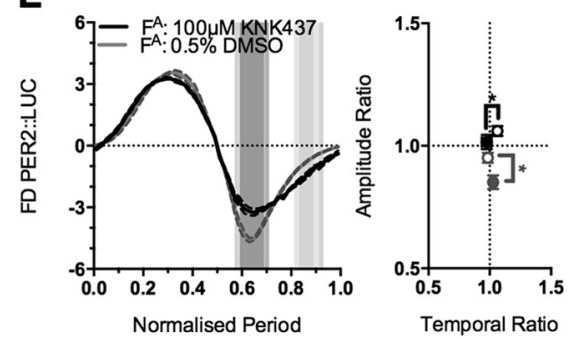


$2 A, B$, top). To test for putative global effects, the profiles were then replotted after normalization to their specific periods (Fig. $2 A, B$, middle), when overlapping plots would represent a global effect of the mutation. This revealed, however, that both mutations produced phase-specific shifts in the standardized waveform that deviated significantly from wild type. Whereas CK1 $\varepsilon^{\text {Tau/Tau }}$ exhibited a relative increase in PER2::LUC early in circadian day compared to wild type, the Fbxl3 ${ }^{A f h / A f h}$ profile exhibited a relative decrease in PER2::LUC later in the circadian day. The effects of the mutations were therefore phase specific rather than global.

To examine these effects more precisely, the first derivative of the period-normalized waveform was calculated, so that the relative rate at which the reporter expression changed over time could be followed quantitatively (Fig. $2 A-C$, bottom). FDA of the wild-type PER2::LUC waveform revealed that circadian cycling of PER2 followed a specific, general pattern of accumulation and decline that is reproducible over the whole cycle (Fig. $2 A-C$, bottom). The pattern of accumulation and decline was significantly different in the mutant waveforms, compared to wild type (Fig. $2 A, B$, bottom). These shifts in FDA suggest that circadian time is encoded as a series of progressive phases, with differential genetic susceptibility, i.e., period changes arising from single genetic manipulations alter the dynamics of these phases selectively, perturbing the resultant profile away from the wild-type trajectory.

\section{Alterations in waveform profile indicate phase-specific points of sensitivity within the oscillator}

As FDA measures how bioluminescence changes over time, by inference it can be used to identify critical intervals where pharmacological manipulation alters peak rates of PER2 accumulation or dissipation. This was exploited across all combinations of genotype and pharmacological manipulation. Shifts in the amplitude and the temporal positions of the maximal rate of increase or decrease in PER2 levels can be expressed as two separate

\section{$\leftarrow$}

Figure 2. Representative single peaks demonstrate alterations in waveform profile caused by genetic manipulation of explant SCN period. $\boldsymbol{A}-\boldsymbol{C}$, Top panels show composite single normalized cycles (solid black) peak aligned and overlaid with wild-type PER2::LUC traces (WT; dashed gray). The top $x$-axis displays time in hours for the wild-type PER2::LUC trace, and the bottom $x$-axis displays time in hours for the PER2::LUC trace of the aligned condition. Central panels display peak-aligned traces as in the top panel on a normalized time base (normalized period). Bottom panels display mean waveform profiles as first derivative of normalized bioluminescence (FD PER2::LUC) versus the normalized period as wild-type profile (solid gray) overlaid with period mutants (solid black). $A, C$, $1 \varepsilon^{\text {Tau/Tau }} \times$ PER2::LUC $\left(C^{\top}\right) . B, F b x / 3^{A f h / A f h} \times$ PER2::LUC ( $\left.F^{A}\right)$. C, Wild-type PER2::LUC slices (WT) treated with vehicle, as follows: baseline (dashed black; top only), $0.1 \%$ DMSO (solid light gray), $0.01 \% \mathrm{H}_{2} \mathrm{O}$ (solid black), and $0.5 \%$ DMSO (solid dark gray). $D-L$, Left, Mean first derivative plot of vehicle-treated (solid gray) or periodaltering-compound-treated (solid black) normalized PER2::LUC bioluminescence (FD PER2::LUC). Right, Constellation plots showing mean shifts in peaks of PER2 accumulation (black) and dissipation (gray). Hollow symbols indicate vehicle treated values, and solid symbols indicate drug-treated values. Values are shown as mean \pm SEM in both $x$ (temporal ratio) and $y$ (amplitude ratio) directions, and significance is indicated by square brackets for either accumulation (black) or dissipation (gray). Treatments are shown on different genetic backgrounds: wild-type PER2::LUC (D-F), $100 \mu \mathrm{m}$ picrotoxin/0.1\% DMSO (D), $1 \mu \mathrm{M}$ PF-670462/ $0.01 \% \mathrm{H}_{2} \mathrm{O}(\boldsymbol{E}), 100 \mu \mathrm{M}$ KNK/0.5\% DMSO $(\boldsymbol{F}) ;\left(K 1 \varepsilon^{\text {Tau/Tau }} \times\right.$ PER2::LUC $(\boldsymbol{G}-\boldsymbol{I}), 100 \mu \mathrm{M}$ picrotoxin/0.1\% DMSO (G), $1 \mu \mathrm{M}$ PF-670462/0.01\% $\mathrm{H}_{2} \mathrm{O}(\boldsymbol{H}), 100 \mu \mathrm{M}$ KNK/0.5\% DMSO (I); Fbx/3 ${ }^{\text {Afh/Afh }} \times$ PER2::LUC (J-L), $100 \mu \mathrm{m}$ picrotoxin/0.1\% DMSO (J), $1 \mu \mathrm{M}$ PF-670462/0.01\% $\mathrm{H}_{2} \mathrm{O}(\boldsymbol{K}), 100 \mu \mathrm{M} \mathrm{KNK} / 0.5 \%$ DMSO $(\boldsymbol{L})$. First derivative plots and alignments on a normalized time base are shown as mean \pm SEM as error banding. For normalized period-aligned plots, gray shading indicates the level of significant difference as assessed by two-way ANOVA, graded by lightest $(p<0.05)$ to darkest ( $p<0.0001$ ), as indicated in the key above $\boldsymbol{A}$. $n$ values are detailed throughout the text. ${ }^{*} p<0.05,{ }^{* *} p<0.01,{ }^{* * *} p<0.001,{ }^{* * * *} p<0.0001$. ratios (one for each parameter) between treatment and baseline (Fig. 2D-L, right). First, however, to ensure that the waveform arrangement is not altered by treatment with vehicle, the three different vehicle treatments were coplotted and aligned on a solar or normalized time base and as FDA plots (Fig. 2C). This revealed no significant difference (two-way ANOVA) arising either from vehicles $\left(0.1 \%\right.$ DMSO vs $0.01 \% \mathrm{H}_{2} \mathrm{O}$ vs $\left.0.5 \% \mathrm{DMSO}, p=0.17\right)$ or from interaction between time and vehicles $(0.1 \%$ DMSO vs $0.01 \% \mathrm{H}_{2} \mathrm{O}$ vs $0.5 \%$ DMSO, $\left.p=0.59\right)$. As there were no significant changes in waveform induced by vehicle treatment, all subsequent comparisons of waveform were made between vehicle and drug.

Picrotoxin reduced the peak amplitude of PER2 accumulation across genotypes compared to vehicle (Fig. $2 D, G$, J; all vs DMSO, wild type, $p<0.01, n=8 / 8$; CK1 $\varepsilon^{\text {Tau/Tau }}, p<0.01, n=$ $\left.8 / 8 ; F b x l 3^{A f h / A f h}, p<0.01, n=8 / 8\right)$. The effect increased as the underlying genetic period increased, with the $F b x l 3^{A f h / A f h}$ mutant producing the largest reduction in amplitude (Fig. $2 J$ ). Across all genotypes, this amplitude effect was associated with an earlier position of the accumulation peak (Fig. 2D, G,J; all vs DMSO, wild type, $p<0.01, n=8 / 8$; CK1 $\varepsilon^{\text {Tau/Tau }}, p<0.01, n=8 / 8$; $\left.F b x l 3^{A f h / A f h}, p<0.02, n=8 / 8\right)$, although it should be noted that the decrease in temporal position seen in the Fbxl $3^{A f h / A f h}$ was very subtle. Picrotoxin also decreased the peak amplitude of dissipation across genotypes (Fig. $2 D, G$,J; all vs DMSO, wild type, $p=$ $0.031, n=8 / 8 ; C K 1 \varepsilon^{\text {Tau/Tau }}, p=0.0005, n=8 / 8 ; F_{b x l 3^{\text {Afh } / A f h}, p<}$ $0.01, n=8 / 8$ ), but without shifting its temporal position. These results suggest that picrotoxin exerts a general effect of reducing the amplitude of peak PER2 accumulation and pushing it earlier in the cycle, whereas PER2 dissipation occurs at the same temporal position but again with a reduced peak rate.

PF-670462 increased the amplitude of the peak rate of accumulation across wild-type and $F b x l 3^{A f h / A f h}$ slices (Fig. $2 E, K$; all vs water, wild type, $p=0.03, n=8 / 8 ; F b x l 3^{A f h / A f h}, p<0.01, n=$ $8 / 8$ ). The effect was not significant in $C K 1 \varepsilon^{\text {Tau/Tau }}$ slices (Fig. $2 H$; PF-670462 vs water, $p=0.12, n=8 / 8$ ). PF-670462 did not affect the temporal position of the accumulation peak in either the wild type or CK1 $\varepsilon^{\text {Tau/Tau }}$ conditions (Fig. $2 E, H$ ), but did delay the accumulation peak of $F b x l 3^{A f h / A f h}$ (Fig. $2 K$; PF-670462 vs water, $p=0.0002, n=8 / 8)$. Across genotypes, PF-670462 caused a reduction in amplitude of the peak PER2 dissipation rate (Fig. $2 E, H, K$; PF-670462 vs water, wild type, $p<0.04, n=8 / 8$; CK1 $\varepsilon^{\text {Tau/Tau }}, p<0.01, n=8 / 8 ; F b x l 3^{\text {Afh/Afh }}, p<0.01, n=8 / 8$ ), but there was no significant effect on the timing of the peak (Fig. $2 E, H, K)$. Thus, PF-670462 generally increases the rate of PER2 accumulation and decreases the subsequent dissipation of PER2 levels, consistent with the idea that PF-670462-mediated inhibition of CK $1 \varepsilon / \delta$ slows down the clock by sustaining elevated levels of PER2 protein by inhibiting its degradation (Lee et al., 2009; Meng et al., 2010).

The HSF1 inhibitor KNK47 caused an earlier peak rate of PER2 accumulation across all genotypes (Fig. $2 F, I, L$; all vs DMSO, wild type, $p<0.01, n=8 / 8$; CK1 $1 \varepsilon^{\text {Tau/Ta }}, p<0.01, n=$ $\left.8 / 8 ; F b x l 3^{A f h / A f h}, p=0.024, n=8 / 8\right)$. In the wild-type SCNs, this shift was associated with an increase in the rate of PER2 accumulation (Fig. 2F; $p<0.01, n=8 / 8$ ) that was absent in the CK1 $\varepsilon^{\text {Tau/Tau }}$ and Fbxl3 ${ }^{A f h / A f h}$ mutants (Fig. 2I,L). Across all genotypes, the peak amplitude of PER2 dissipation rate was also reduced (Fig. $2 F, I, L$; all vs DMSO, wild type, $p<0.01, n=8 / 8$; CK1 $\varepsilon^{\text {Tau/Tau }}, p<0.01, n=8 / 8 ;$ Fbxl3 $3^{\text {Afh/Afh }}, p=0.012, n=8 / 8$ ), without any effect on the timing of the peak. These results suggest that KNK437 treatment shifts PER2 accumulation to an earlier phase of the cycle without affecting the amplitude of the peak rate 
of accumulation. This early burst of PER2 accumulation combined with the severe reduction of its amplitude could extend the SCN period because PER2 takes longer to accumulate to a physiologically relevant level for clock progression.

FDA therefore indicated a shared general effect of the periodaltering drugs on the peaks of the waveform profile regardless of genetic background. Nevertheless, while the direction of the change is dictated principally by pharmacology, the degree of difference in the peak rate of PER2 accumulation or dissipation was again dictated by the interaction between pharmacology and genetics. These shifts in PER2 dynamics thereby indicate sensitive phases or "checkpoints" in the circadian cycle that can be differentially probed through interacting genetic and pharmacological manipulations.

\section{Pharmacological manipulation of the SCN clockwork has a time-stamped phase effect regardless of underlying genotype}

To further explore and characterize the phase-specific changes to the circadian oscillation, FDA during baseline was subtracted from the corresponding FDA during drug treatment (Fig. 3). This would cancel out any genotypic effect and thereby reveal any specific drug effect. FDA-S was validated using vehicle treatment, where there should be little difference between the baseline and treatment curves, and indeed this was the case across all genotypes (two-way ANOVA, 0.1\% DMSO vs $0.01 \% \mathrm{H}_{2} \mathrm{O}$ vs $0.5 \%$ DMSO, CK1 $\varepsilon^{\text {Tau/Tau }}, p>0.99$; wild type, $p>0.99 ; \mathrm{Fbxl} 3^{\text {Afh/Afh }}$, $p>0.99)$ and between the different genotypes within vehicle treatment (CK1 $\varepsilon^{\text {Tau/Tau }}$ vs wild type vs Fbxl3 ${ }^{\text {Afh/Afh }}, 0.1 \%$ DMSO, $\left.p=0.99 ; 0.01 \% \mathrm{H}_{2} \mathrm{O}, p=0.95 ; 0.5 \% \mathrm{DMSO}, p=0.99\right)$. Thus, FDA-S was used to identify general phase-specific patterning across the cycle in response to pharmacological manipulation.

Picrotoxin FDA-S revealed a general time-stamped patterning of sensitive phases to treatment regardless of genetic background (Fig. $3 A, D, G, J)$. These phases are highlighted by peaks in the FDA-S profile at which there were significant differences between vehicle and treatment. Interestingly, in the picrotoxin-treated CK1 $\varepsilon^{\text {Tau/Tau }}$ FDA profile comparison, the vehicle-treated FDA profile appears to mask low-amplitude changes in the picrotoxintreated waveform (Fig. $2 G$ ) that are revealed in the FDA-S profile at two points (Fig. 3D): toward the start of the cycle and just after the PER2 peak. Thus, FDA-S analysis was able to unmask changes in the profile undetected by the FDA approach, adding sensitivity to this analytical approach. In addition to this unmasking of sensitive phases, FDA-S revealed that the pattern of phase-specific pharmacological sensitivity across genotypes followed the same general trend in directionality, and that the temporal position of the peaks coincided in circadian time (Fig. $3 J, M$ ). The exception to this lay in the case of the final peak of $C K 1 \varepsilon^{\text {Tau/Tau }}$, where the amplitude of the difference was too low to register as significant when the picrotoxin profile was compared to the vehicle profile (Fig. 3D). However, automated peak identification enabled the mapping of this peak, which registered with the phase patterning of the other genotypes (Fig. $3 J$ ) and had a severely and significantly reduced amplitude (peak 4 ; CK1 $\varepsilon^{\text {Tau/Tau }}$ vs wild type, $p<0.01$; CK1 $\varepsilon^{\text {Tau/Tau }}$ vs Fbxl3 $3^{A f h / A f h}, p<0.01$; Fig. 3M).

Interestingly, while the phases of the critical points of manipulation were approximately the same across genotypes, the amplitudes of the phase-specific changes were significantly different between different phases and genotypes (Fig. $3 A, D, G, M$ ). If these differences arose solely as a result of the pharmacological treatment, then the amplitude of difference between the three genotypes should not vary. If, however, there were differences between the genotypes, this could arise from a synergistic phase- specific genotype by pharmacology interaction. This synergistic interaction would be most informative when the difference occurred between wild-type and circadian mutants, as this could point to a mechanism where the clockwork is more or less sensitive to a particular manipulation due to genetics. Applying this analysis to $100 \mu \mathrm{M}$ picrotoxin revealed a significant reduction in amplitude between $C K 1 \varepsilon^{\text {Tau/Tau }}$ and wild type during the first two peaks ( $C K 1 \varepsilon^{\text {Tau/Tau }}$ vs wild type, peak $1, p<0.01$; peak $2, p<$ $0.01)$. This indicates that the $C K 1 \varepsilon^{\text {Tau/Tau }}$ mutant is less sensitive to picrotoxin treatment at these particular phases, which resulted in a smaller difference between the genotype and the pharmacological treatment.

As with picrotoxin, FDA-S revealed a unique fingerprint of phase-specific patterning in $1 \mu \mathrm{M}$ PF-670462-treated waveforms (Fig. $3 B, E, H, K$ ). This registered to a high degree across genotypes, with the phases of all four peaks aligning across the cycle (Fig. $3 \mathrm{~K}$ ). Different from the picrotoxin-treated slices, PF-670462 also produced comparable changes in amplitude across the genotypes tested (Fig. $3 N$ ). As there was no significant difference in either amplitude or phase-sensitive patterning across genotypes, this indicates that treatment with 1 $\mu \mathrm{M}$ PF-670462 induced the same changes across genotypes. This could be due to the fact that this compound acts on both the $\mathrm{CK} 1 \varepsilon$ and $\mathrm{CK} 1 \delta$ isoforms, and therefore reverses the effects of the $C K 1 \varepsilon^{T a u / T a u}$ mutation to an approximately wild-type level and affects CK1 $\delta$ to the same degree across genotypes, causing the characteristic period-lengthening effects of this drug (Meng et al., 2010) and clamping the waveform into a particular phase pattern.

FDA-S of KNK437-induced waveforms revealed a general phase-specific patterning comprising four peaks that is visually present at low amplitude across all three genotypes (Fig. 3C, F, I), although it was too low to achieve significance (Fig. $3 L$ ). This lack of phase-specific patterning was lost between the three genotypes at the beginning of the cycle, but was observed with a high degree of registration and significance at the end of the cycle (Fig. $3 C, F, I, L)$. Looking at the waveforms in detail, $C K 1 \varepsilon^{\text {Tau/Tau }}$ highlights the four canonical KNK437 phases that could still be identified across the other two genotypes, albeit at low amplitude (peaks 2-5; Fig. 3F,L). This low amplitude footprint was again mapped by automated peak identification with a high degree of registration when coplotted (Fig. $3 L$ ), but the first two canonical peaks [peaks $2\left(F b x l 3^{A f h / A f h}\right)$ and 3 (wild type and $\left.F b x l 3^{A f h / A f h}\right)$ ] could be distinguished from the vehicle waveform by two-way ANOVA (Fig. 3C, I, L). Automated peak registration revealed that although there was a low amplitude difference at the second and third peaks, there was no significant difference between the genotypes. This suggests the possibility of a functional limit to FDA-S (as the period extreme is reached), where amplitude of the oscillation is reduced and the individual waveforms become too noisy to discriminate by direct waveform comparison. This peak identification approach suggests that the four canonical peaks (peaks 2-5) comprise the central footprint for KNK437 treatment preserved across genotypes (Fig. $3 \mathrm{~L}, \mathrm{O}$ ).

Remarkably, FDA-S revealed that pharmacological treatment generally appears to cause a phase-specific patterning of the waveform, where certain phases are revealed to be more or less sensitive to manipulation. This phase-patterned fingerprint allows the identification of synergistic interactions between genotype and pharmacology and allows the potential identification of cryptic clockwork mechanisms. Thus, the FDA/FDA-S approach reveals that manipulation of period genetically, pharmacologi- 

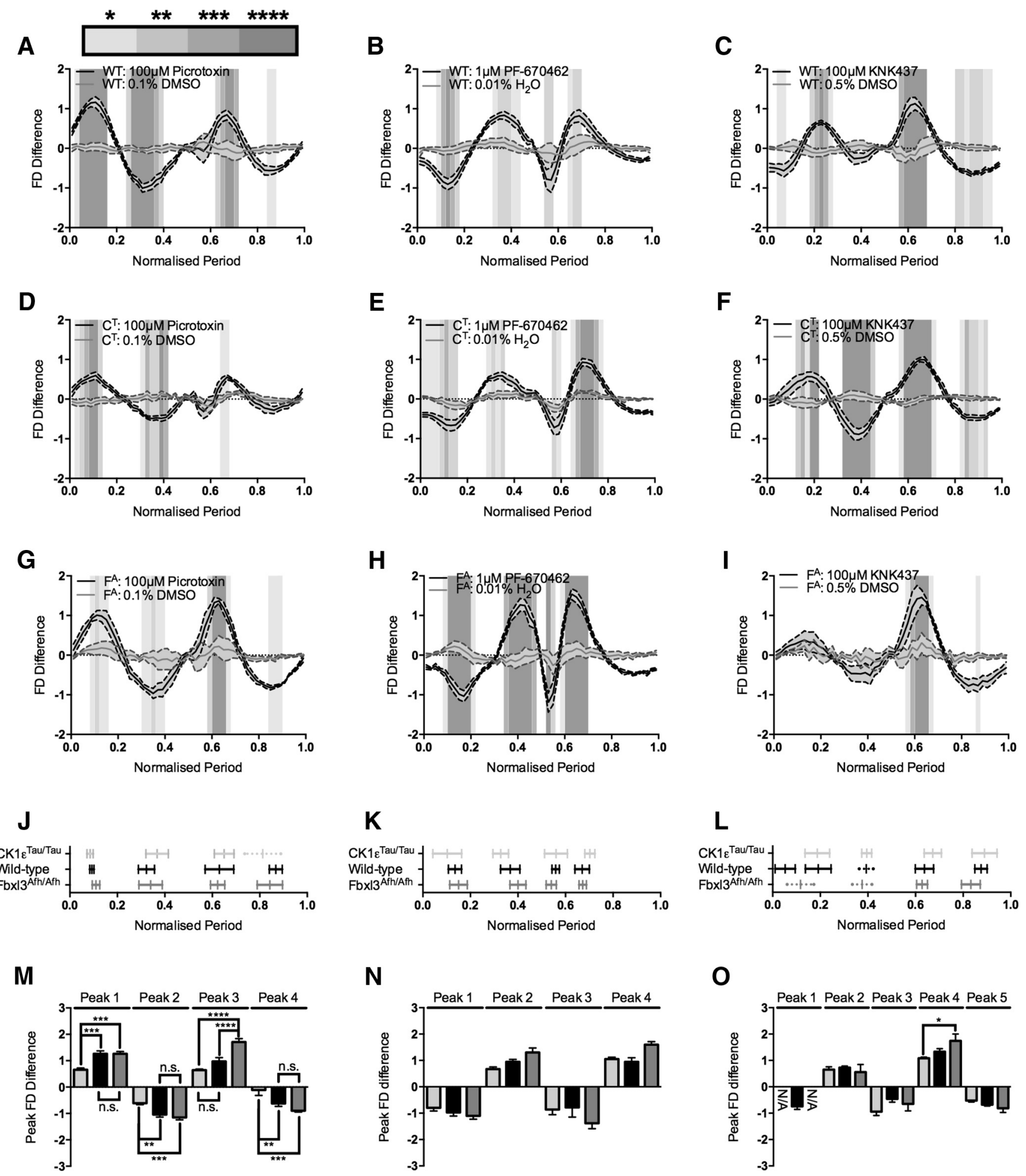

Figure 3. Baseline subtraction (FDA-S) reveals phase-specific pharmacological patterning. $\boldsymbol{A}-\boldsymbol{I}$, Baseline-subtracted treatment cycles (FD Difference) versus normalized period ordered by genotype and treatment. Vehicle (solid gray) and treatment (solid black) are coplotted as mean \pm SEM as shaded error banding. The significant differences between vehicle and treatment determined by two-way ANOVA are indicated by graded gray shading as detailed in the key above $A$. Treatments are as follows: wild-type PER2::LUC (WT; $A-C), 100 \mu \mathrm{m}$ picrotoxin/0.1\% DMSO (A),

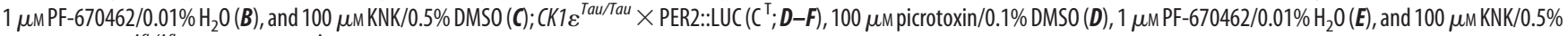

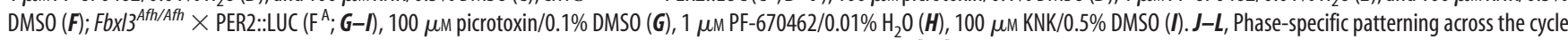
showing the temporal alignment of CK1 $\varepsilon^{\text {Tau/Tau }} \times$ PER2::LUC (light gray), wild-type PER2::LUC (black), and Fbx/3 ${ }^{\text {Afh/Afh }} \times$ PER2::LUC (dark gray) treated with period-altering compounds. Bars show mean peak position \pm SD. Solid-capped bars show phase intervals that are significantly different from vehicle in $A-I$. Dotted uncapped bars show phase intervals that have been identified only via automated peak identification. Treatment and vehicle identities are as follows: $100 \mu \mathrm{m}$ picrotoxin/0.1\% DMSO (J), $1 \mu \mathrm{M}$ PF-670462/0.01\% $\mathrm{H}_{2} \mathrm{O}(\boldsymbol{K})$, and $100 \mu \mathrm{m} \mathrm{KNK437/0.5 \%} \mathrm{DMSO} \mathrm{(L).} \mathrm{M-0,} \mathrm{Peak}$ amplitudes of peak phase patterning intervals (Peak FD Difference) illustrated in $J-L$. Bars are mean \pm SEM and indicate the difference between vehicle and treatment for particular genotypes: $C K 1 \varepsilon^{\text {Tau/Tau }} \times$ PER2::LUC (light gray), wild-type PER2::LUC (black), and Fbx/3 Afh/Afh $\times$ PER2::LUC (dark gray). Significant differences are noted by square brackets. Treatment and vehicle identities are as follows: $100 \mu \mathrm{m}$ picrotoxin/0.1\% DMSO (M), $1 \mu \mathrm{m} \mathrm{PF}-670462 / 0.01 \% \mathrm{H}_{2} \mathrm{O}(\boldsymbol{M})$, and $100 \mu \mathrm{m}$ KNK437/0.5\% DMSO (O). nvalues are detailed throughout the text. ${ }^{*} p<0.05,{ }^{* *} p<0.01,{ }^{* * *} p<0.001,{ }^{* * * *} p<0.0001$. 
cally, or in combination manifests as a change in waveform (Fig. 2) that is not revealed by peak alignment alone. Finally, the FDA-S approach also reveals that pharmacologically induced changes in waveform have a phase-specific pattern of manipulation. The temporal patterning of this is independent of genotype, but the amplitude of the change at sensitive phases represents a genotype by pharmacology interaction (Fig. 3).

\section{Waveform profile does not encode network period, but can reveal phase-specific core clock mechanisms}

To make an independent assessment of the validity of FDA/ FDA-S to test the relationship between period and pharmacologically sensitive phases of the oscillation, CK1 $\varepsilon^{\text {Tau/Tau }}$ SCNs were titrated with a range of doses of the CK1 $\varepsilon$ specific inhibitor PF4800567 (3-[(3-Chlorophenoxy)methyl]-1-(tetrahydro-2 H-pyran4-yl)- $1 H$-pyrazolo[3,4-d]pyrimidin-4-amine hydrochloride) (Fig. 4), a drug that selectively lengthens the period of CK1 $\varepsilon^{\text {Tau/Tau }}$ rhythms with minimal effect on wild-type slices (Meng et al., 2010). Through use of this pharmacological manipulation to oppose a genetic manipulation and reset period, in theory, the wild-type and $C K 1 \varepsilon^{\text {Tau/Tau }} \mathrm{SCNs}$ should exist with identical periods but different genetics. PF-4800567 at $0.5 \mu \mathrm{M}$ drove the $C K 1 \varepsilon^{T a u / T a u}$ slices to the wild-type $24 \mathrm{~h}$ period (Fig. $4 A, B$; baseline, $20.00 \pm 0.10 \mathrm{~h}$ vs $0.5 \mu \mathrm{M}$ PF-4800567, $23.83 \pm$ $0.20 \mathrm{~h} ; p<0.01 ; n=6)$. FDA comparison of $C K 1 \varepsilon^{\text {Tau/Tau }}$ slices treated with $0.5 \mu \mathrm{M}$ PF-4800567 revealed a significant shift in the waveform profile across the cycle (two-way ANOVA; Fig. 4C). As the CK1 $\varepsilon^{\text {Tau/Tau }}$ mutation accelerates the circadian clock through dysregulated phosphorylation of the PER proteins (Lowrey et al., 2000; Meng et al., 2008, 2010; Maywood et al., 2014), pharmacological attenuation of $\mathrm{CK} 1 \varepsilon$ activity should return the CK1 $\varepsilon^{\text {Tau/Tau }}$ waveform to the wild-type profile if waveform is directed solely by period. To test this, two comparisons with the wild-type profile were made. First, comparison of the baseline genetic waveform (CK1 $\varepsilon^{\text {Tau/Tau }}$ vs wild type; Fig. $\left.4 D\right)$ showed that the baseline profiles were significantly different in broadly the same phases as $C K 1 \varepsilon^{\text {Tau/Tau }}$ baseline and $C K 1 \varepsilon^{\text {Tau/Tau }} 0.5 \mu \mathrm{M}$ PF-4800567 (Fig. 4C). Second, comparison between the wildtype baseline and $C K 1 \varepsilon^{\text {Tau/Tau }} 0.5 \mu \mathrm{M}$ PF-4800567-treated profiles (Fig. 4E) revealed that this dose of PF-4800567 partially reversed the $C K 1 \varepsilon^{T a u / T a u}$ mutation, but still left a significant mismatch between profiles in the latter half of the cycle. This indicates that the CK1 $1 \varepsilon^{\text {Tau/Tau }}$ mutation is sensitive to pharmacological inhibition in the first half of the cycle but not in the second half. From this, a reasonable conclusion is that the CK1 $\varepsilon^{\text {Tau/Tau }}$ mutation exerts influence over the period of the oscillation through inappropriately phased activity at the start of the cycle.

The remaining mismatches between wild-type and CK1 $\varepsilon^{\text {Tau/Tau }}$ slices treated with $0.5 \mu \mathrm{M}$ PF-4800567 suggested two things. Either waveform profile is not a product of the period expressed, or returning the SCN clock to wild-type levels pharmacologically is more complicated than simply manipulating the period, i.e., there is a significant interaction between genotype and pharmacology that established circadian analyses do not reveal. To investigate this interaction further, wild-type slices were treated with the same dose of PF-4800567 as the CK1 $\varepsilon^{\text {Tau/Tau }}$ slices (Fig. $4 B, F$ ). This caused a small but significant increase in period (Fig. $4 B$; baseline, $24.61 \pm 0.07 \mathrm{~h}$ vs $0.5 \mu \mathrm{M}$ PF-4800567, $25.65 \pm 0.12 \mathrm{~h} ; p=0.002 ; n=5$ ), which differs from previous reports that PF-4800567 is ineffective on wild-type SCNs (Meng et al., 2010; Pilorz et al., 2014). Closer analysis of the waveform profile showed that there was a significant mismatch between baseline and $0.5 \mu \mathrm{M}$ PF-4800567-treated profiles (Fig. $4 F$ ) that broadly occurred in the same phases as the mismatch between wild-type baseline and $C K 1 \varepsilon^{\text {Tau/Tau }}$ treated with $0.5 \mu \mathrm{M}$ PF4800567 (Fig. 4E). Due to the persistent mismatch in the latter half of the cycle in both treated conditions, these conditions were compared in a coplotted FDA, which showed that the profiles became completely aligned with no significant difference (Fig. $4 G$ ). These results revealed differential phases of regulation for the wild-type and Tau versions of the CK1 $\varepsilon$ enzyme, where the wild-type version predominately directs PER2 destabilization in the latter half of the cycle (Fig. $4 F$ ), while the Tau mutant becomes active at the beginning of the cycle, and it is presumably this "inappropriately phased" gain-of-function activity that causes the marked period acceleration (Fig. 4B).

This period mismatch was further backed-up by the FDA-S analysis (Fig. 3). This revealed that there were large shifts in the waveform in the first three quarters of the cycle for $C K 1 \varepsilon^{\text {Tau/Tau }}$ slices treated with PF-4800567 that were absent from the wildtype slices (Fig. $4 H-K ; C K 1 \varepsilon^{\text {Tau/Tau }}$ vs wild type, peak $1, p<0.01$, $n=6 / 5$; peak $2, p<0.01, n=6 / 5$; peak $3, p<0.01, n=6 / 5)$. The final sensitive phase of the cycle had a shifted temporal positioning between $C K 1 \varepsilon^{T a u / T a u}$ and wild type (CK1 $\varepsilon^{\text {Tau/Tau }}$ vs wild type, peak $4, p=0.01, n=6 / 5)$, but did not show any significant difference in magnitude (Fig. $4 J, K ; C K 1 \varepsilon^{\text {Tau/Tau }}$ vs wild type, peak $4, p=0.11, n=6 / 5$ ). Due to the very specific action of this drug on the CK1 $\varepsilon$ isoforms (Walton et al., 2009), the FDA-S also revealed an expected genotype and pharmacology interaction in $C K 1 \varepsilon^{\text {Tau/Tau }}$ treated slices, which is especially evident when compared to the non-responsive patterning of the wild-type treated slices (Fig. $4 J, K$ ). Together, these results indicate that the first derivative analysis is a useful tool for interrogating clock function mechanistically across the cycle and that FDA-S reveals not only pharmacological phase-specific patterning, but also significant internal phases where genotype and pharmacology interact.

\section{Network and cell-autonomous properties of the SCN are maintained at extreme periods}

Bioluminescence recorded by PMT is the integrated product of signal across the SCN slice. At extreme periods, this may or may not mask alterations to circuit-level function. To explore the relationship between changes in waveform and period in the context of cell-autonomous and circuit-level properties of the SCN, slices oscillating at the two extreme periods were visualized directly by CCD camera to achieve cellular resolution of bioluminescence (Fig. 5). SARFIA analytical routines identified individual oscillators (presumed cells) across the network. In $C K 1 \varepsilon^{\text {Tau/Tau }}$ slices treated with $100 \mu \mathrm{M}$ picrotoxin, individual oscillators maintained the characteristic pharmacologically determined period of the aggregate signal (Fig. 5A; baseline vs treatment, $p<0.01, n=4$ ) and remained in phase alignment with each other as assessed by raster plots and Rayleigh analysis (Fig. $5 B, C$; mean vector length, baseline vs treatment, $p=0.53, n=4)$. In $F b x l 3^{A f h / A f h}$ slices treated with $100 \mu \mathrm{M}$ KNK437, individual oscillators still sustained the extreme long period of the aggregate signal (Fig. $5 D$; baseline vs treatment, $p<0.01, n=4$ ) and maintained robust synchrony across the network (Fig. $5 E, F$; mean vector length: baseline vs treatment, $p=0.83, n=4)$. Thus, the ability of the SCN to sustain extremely long or extremely short circadian periods is an integral property of the circuit, and the mechanisms that mediate synchrony can operate effectively over a wide range of nontypical periods that extends at least between 17 and $46 \mathrm{~h}$. Importantly, the changes in waveform that accom- 
A

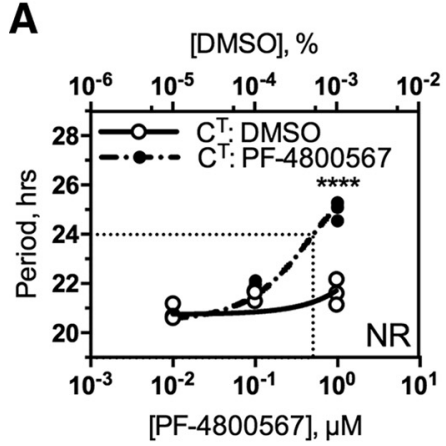

D

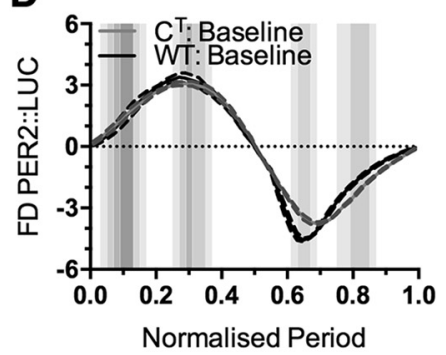

G

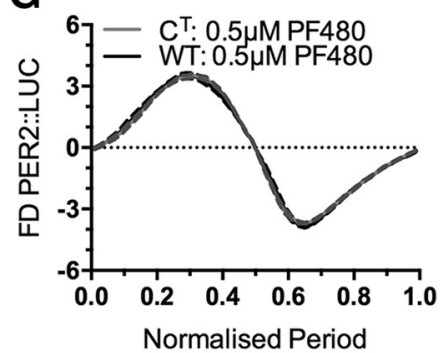

J

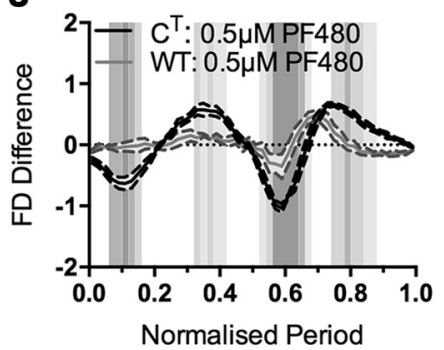

B ㅁ Baseline

$0.5 \mu \mathrm{M}$ PF-4800567

Washout

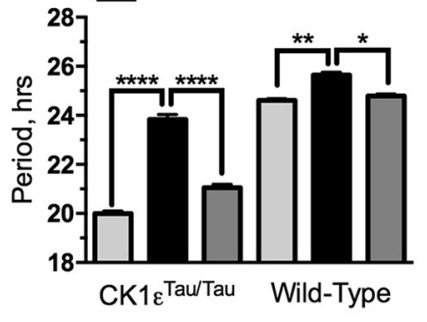

$E$

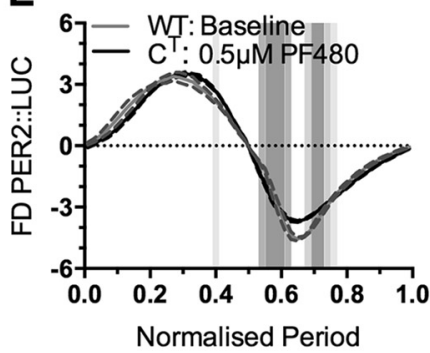

$\mathrm{H}$

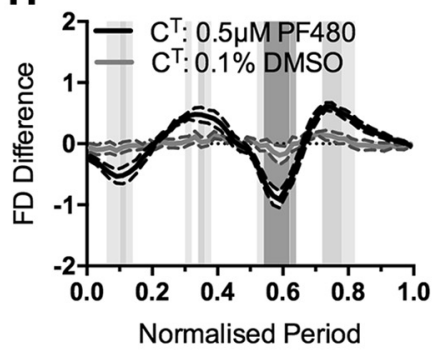

K

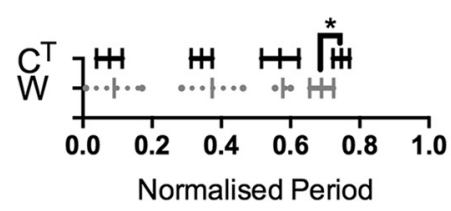

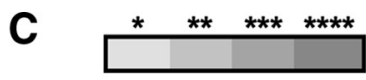

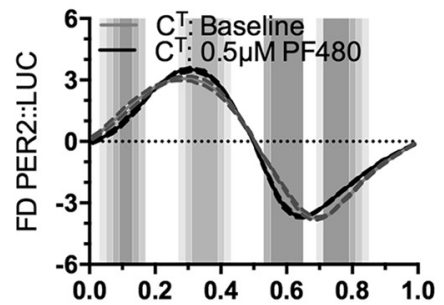

Normalised Period

F

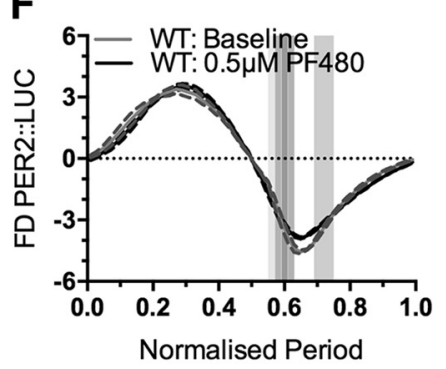

I
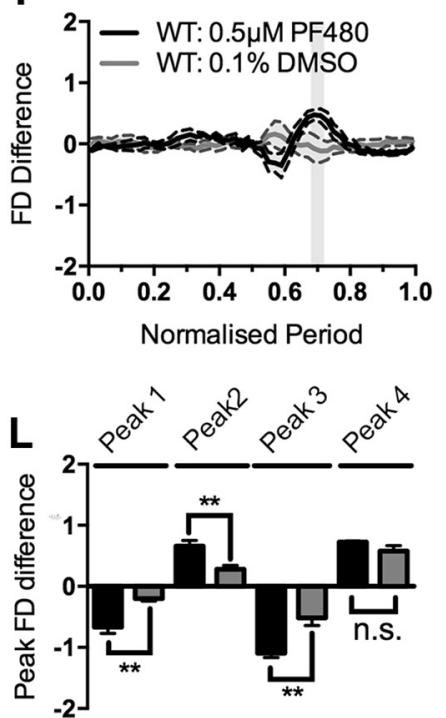

Figure 4. First derivative plots reveal differential sensitivity of genotypes to pharmacological manipulation. A. Dose-response curve of CK1 $\varepsilon^{\text {Tau/Tau }} \times$ PER2::LUC genotype titrated with increasing concentrations of the CK1 $\varepsilon$ inhibitor PF-4800567 (solid black circles) and DMSO (hollow circles). The critical dose to return CK1 $\varepsilon^{\text {Tau/Tau }} \times$ PER2::LUC to the wild-type period is indicated by the dotted line. NR indicates that at $10 \mu \mathrm{M}$, slices become nonrhythmic. $\boldsymbol{B}$, Summary period data for baseline (light gray), $0.5 \mu \mathrm{M}$ PF- 4800567 (black), and washout (dark gray) in CK1 $\varepsilon^{\text {Tau/Tau }} \times$ PER2::LUC slices and wild-type PER2::LUC slices. Genotype is indicated below the bars. Values are shown as mean \pm SEM. C $-\mathbf{G}$, First derivative of normalized bioluminescence (FD PER2::LUC) versus the normalized period showing CK1 $\varepsilon^{\text {Tau/Tau }} \times$ PER2:::LUC treated with $0.5 \mu \mathrm{m}$ PF-4800567 (C $: 0.5 \mu \mathrm{m}$ PF480; $C$, black) overlaid with CK1 $\varepsilon^{\text {Tau/Tau }}$ baseline ( $C^{\top}$ : Baseline; gray), wild-type PER2::LUC baseline (WT: Baseline; $\boldsymbol{D}$, black) overlaid with CK1 $\varepsilon^{\text {Tau/Tau }} \times$ PER2::LUC baseline (C': Baseline; gray), CK1 $\varepsilon^{\text {Tau/Tau }} \times$ PER2::LUC treated with $0.5 \mu \mathrm{M}$ PF-4800567 ( ( ${ }^{\top}: 0.5 \mu \mathrm{M}$ PF480; $E$, black) overlaid with wild-type PER2::LUC baseline (WT: Baseline; gray), wild-type PER2::LUC treated with $0.5 \mu \mathrm{M}$ PF-4800567 (WT: $0.5 \mu \mathrm{M}$ PF480; $\boldsymbol{F}$, black) overlaid with wild-type baseline (WT: Baseline; gray), and CK1 $\varepsilon^{\text {Tau/Tau }} \times$ PER2::LUC treated with $0.5 \mu \mathrm{M}$ PF-4800567 (C ${ }^{\top}$ : PF480; $\boldsymbol{G}$, black) overlaid with wild-type PER2::LUC treated with $0.5 \mu \mathrm{m}$ PF-4800567 (WT: PF480; gray). Values are mean \pm SEM, indicated by shaded error banding. $\boldsymbol{H}$-J, Baseline subtraction showing (FD Difference) CK1 $\varepsilon^{\text {Tau/Tau }} \times$ PER2::LUC treated with either $0.5 \mu \mathrm{M}$ PF-4800567 (black) or 0.1\% DMSO (gray; $\boldsymbol{H}$ ), wild-type PER2::LUC treated with either $0.5 \mu \mathrm{M}$ PF-4800567 (black) or $0.1 \%$ DMSO (gray; $\boldsymbol{l}$ ), and $C K 1 \varepsilon^{\text {Tau/Tau }} \times$ PER2::LUC treated with $0.5 \mu \mathrm{M}$ PF-4800567 (black) overlaid with wild-type PER2::LUC treated with $0.5 \mu \mathrm{m}$ PF-4800567 (gray; J). Values are mean \pm SEM, indicated as shaded error banding. $\boldsymbol{K}$, Phase-specific patterning across the cycle showing the temporal alignment of CK1 $\varepsilon^{\text {Tau/Tau }} \times$ PER2::LUC ( $C^{\top}$; black) and wild-type PER2::LUC (WT; gray) peaks from slices treated with $0.5 \mu \mathrm{M}$ PF-4800567. Bars show mean peak position \pm SD. Solid capped bars show phase intervals that are significantly different from vehicle in $\boldsymbol{H}$ and $\boldsymbol{I}$. Dotted uncapped bars show phase intervals that have been identified only via automated peak identification. $L$, Peak amplitudes of peak phase patterning intervals highlighted in $\boldsymbol{K}$. Bars are mean \pm SEM and indicate the absolute amplitude for either CK1 $\varepsilon^{\text {Tau/Tau }} \times$ PER2::LUC (black) or wild-type PER2::LUC (gray). Significant differences are noted by square brackets. Significant differences for first derivative plots $(\mathbf{C}-\mathbf{G})$ and baseline subtractions $(\mathbf{G}-\boldsymbol{J})$ are indicated by graded gray shading as described in the key in $\mathbf{C} . n$ values are detailed throughout the text. ${ }^{*} p<0.05,{ }^{* *} p<0.01$, ${ }^{* * *} p<0.001,{ }^{* * * *} p<0.0001$. 
A
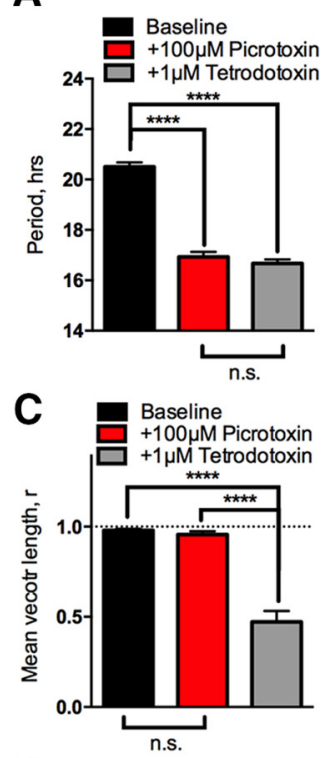

D
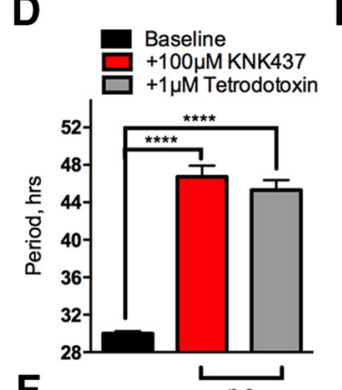

$\mathbf{F}$

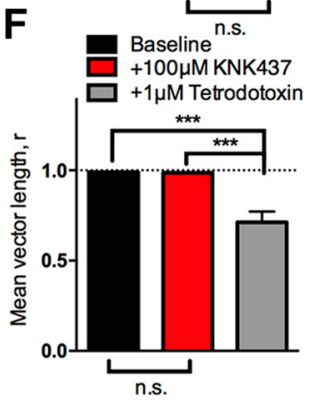

B
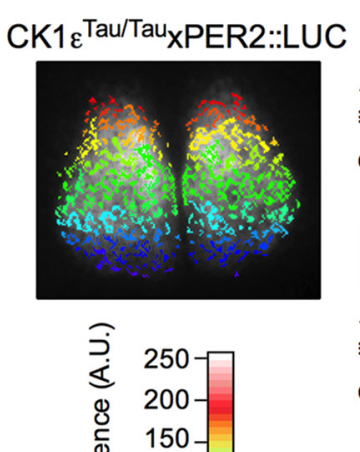
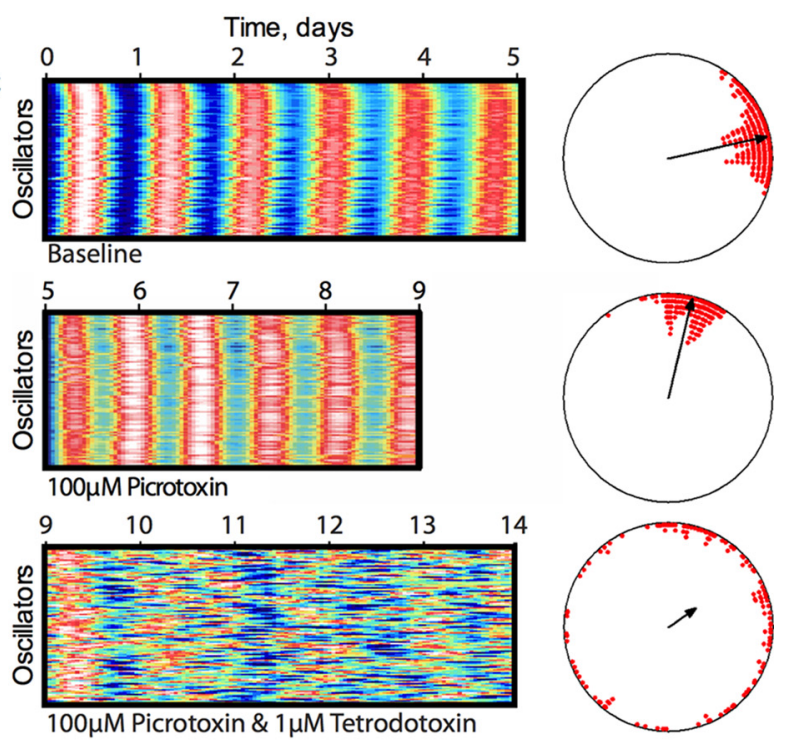

Time, days

E

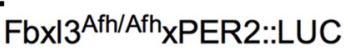
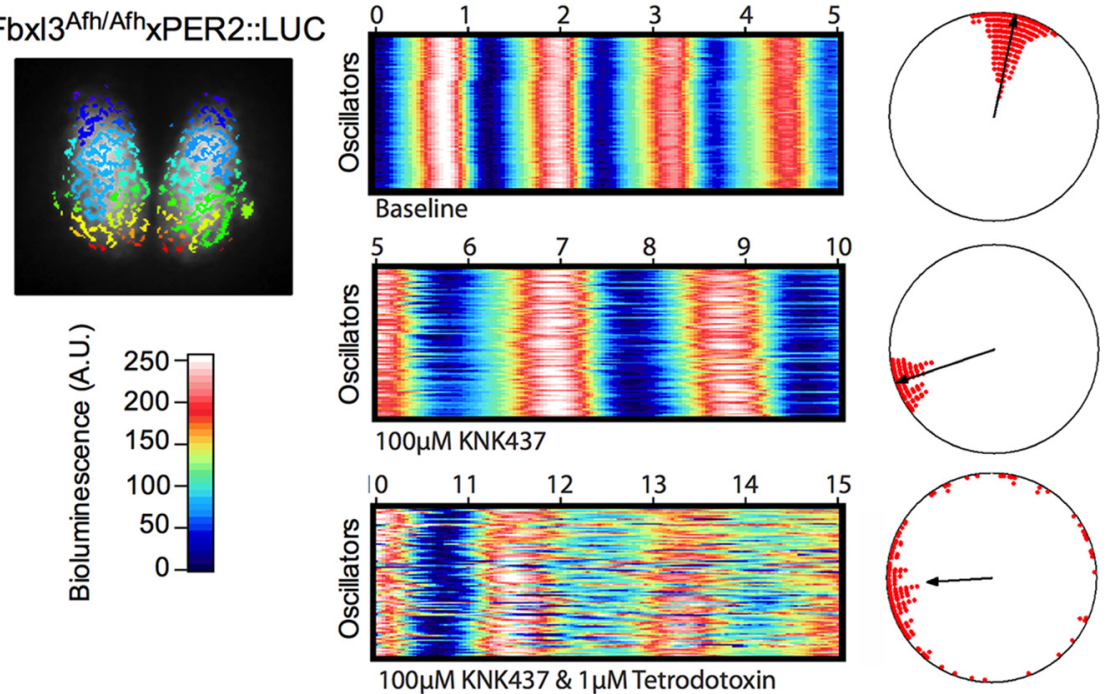

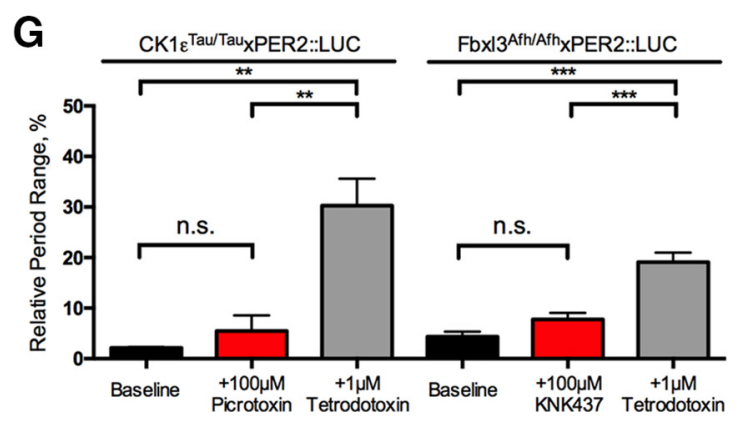

Figure 5. Network and cell-autonomous properties of the $S C N$ are unaffected by pushing period to short or long extremes. $A-F$, SARFIA single-cell imaging analysis of $C K 1 \varepsilon^{\text {Tau/Tau }} \times P$ PER2::LUC

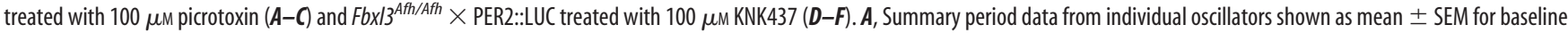
(black), $100 \mu \mathrm{m}$ picrotoxin (+100 $\mu \mathrm{m}$ picrotoxin; red), and $100 \mu \mathrm{m}$ picrotoxin/1 $\mu \mathrm{m} \mathrm{TTX} \mathrm{cotreatment} \mathrm{(}+1 \mu \mathrm{m}$ TTX; gray). B, Individual oscillators were assessed by SARFIA identification and analysis of ROIs, indicated as color-coded regions on the inset bioluminescent SCN image (left). Raster plots of 100 individual representative oscillators within the SCN are shown for baseline (top), $100 \mu \mathrm{M}$ picrotoxin (middle), and $100 \mu \mathrm{m}$ picrotoxin/1 $\mu \mathrm{m}$ TTX cotreatment (bottom). Relative bioluminescence intensity is color coded according to the color bar. Rayleigh plots are shown next to their corresponding raster plot. Phases of individual oscillators are plotted as circles, and the degree of synchrony is indicated by the length of the vector in the center. C, Summary synchrony data reported as mean vector length from individual Rayleigh analyses. Values are shown as mean \pm SEM for baseline (black), $100 \mu \mathrm{m}$ picrotoxin (red), and $100 \mu \mathrm{m}$ picrotoxin/1 $\mu \mathrm{m}$ TTX cotreatment (gray). $\boldsymbol{D}$, Summary period data from individual oscillators shown as mean \pm SEM for baseline (black), $100 \mu \mathrm{m} \mathrm{KNK437} \mathrm{(+100} \mu \mathrm{m} \mathrm{KNK437;} \mathrm{red),} \mathrm{and} 100 \mu \mathrm{m} \mathrm{KNK437/1} \mu \mathrm{M} \mathrm{TTX} \mathrm{cotreatment} \mathrm{(+1} \mu \mathrm{m} \mathrm{TTX;}$ gray). $\boldsymbol{E}$, Individual oscillators were assessed by SARFIA identification and analysis of ROIs, indicated as color-coded regions on the inset bioluminescent SCN image (left). Raster plots of 100 individual representative oscillators within the $S C N$ are shown for baseline (top), $100 \mu \mathrm{m}$ picrotoxin (middle), and $100 \mu \mathrm{m}$ KNK437/1 $\mu \mathrm{M}$ TTX cotreatment (bottom). Relative bioluminescence intensity is color coded according to the color bar. Rayleigh plots shown next to their corresponding raster plot. Phases of individual oscillators are plotted as circles, and the (Figure legend continues.) 
pany divergent periods are not a consequence of changes in phase synchrony across the network.

To determine the relative contributions of cell-autonomous and circuit-level mechanisms to the maintenance of extreme periods in synchronized SCN, TTX was added during pharmacological treatment. TTX uncouples the SCN network by blocking action potential firing, leading to progressively damped and desynchronized SCN cellular oscillations (Yamaguchi et al., 2003; Hastings et al., 2007). In CK1 $\varepsilon^{\text {Tau/Tau }}$ SCN treated with $100 \mu \mathrm{M}$ picrotoxin (Fig. 5A-C), individual cells lost phase coherence when treated with TTX (Rayleigh mean vector, $100 \mu \mathrm{M}$ picrotoxin alone vs with $1 \mu \mathrm{M}$ TTX, $p<0.01, n=4)$. Moreover, the coherence of individual cellular rhythms as assessed by the RAE was reduced by TTX ( $100 \mu \mathrm{M}$ picrotoxin, alone, $0.08 \pm 0.01$ vs 1 $\mu \mathrm{M}$ TTX, $0.34 \pm 0.10 ; p=0.03 ; n=4)$. Nevertheless, the overall cellular period was not significantly different from that of SCNs without TTX $(100 \mu \mathrm{M}$ picrotoxin alone vs with $1 \mu \mathrm{M}$ TTX, $p=$ $0.34, n=4$ ), demonstrating that individual SCN cells are able to sustain sub-17 h circadian periods, and even with this short period, circuit-level mechanisms are able to maintain synchronization. In the complementary, extremely long-period condition, Fbxl $3^{\text {Afh/Afh }}$ SCNs treated with $100 \mu \mathrm{M}$ KNK437 and also treated with TTX (Fig. 5D-F) exhibited desynchronization of the circuit (Rayleigh mean vector, $100 \mu \mathrm{M}$ KNK437 alone vs with $1 \mu \mathrm{M}$ TTX, $p<0.01, n=4)$ and reduction of the coherence of individual cellular rhythms (100 $\mu \mathrm{M}$ KNK437 alone 0.13 vs with $1 \mu \mathrm{M}$ TTX, $0.22 \pm 0.06, p=0.03, n=4)$. As with the short-period condition, the overall cellular period was not significantly different from that of the SCN without TTX (100 $\mu \mathrm{M}$ KNK437 alone vs with 1 $\mu \mathrm{M}$ TTX, $p=0.31, n=4$ ), demonstrating that individual SCN cells are also able to sustain autonomous circadian periods of over $42 \mathrm{~h}$.

SCN explant cultures express a high degree of precision and coherence between individual oscillators due to the strong coupling properties of the network. If, however, this coupling is disrupted (e.g., by dispersal), these oscillators become comparatively more imprecise (Herzog et al., 2004). To determine whether pushing the period to these extremes compromises the precision of the intact network, the range of periods revealed by individual oscillators (Fig. $5 A, D$ ) was calculated and expressed as a percentage of the $S C N$ aggregate period (Fig. $5 G$ ). In the baseline condition, neither mutation produced a significant deviation from the relative period range of wild-type SCNs (data not shown; wild type vs $C K 1 \varepsilon^{\text {Tau/Tau }}$ vs Fbxl3 $3^{\text {Afh/Afh }}, 2.74 \pm 0.38 \%$ vs $2.14 \pm 0.15 \%$ vs $4.36 \pm 1.00 \% ; p=0.08 ; n=4 / 4 / 4)$. At the short-period extreme, the precision and coherence of the oscillation were maintained $\left(C K 1 \varepsilon^{\text {Tau/Tau }}\right.$ baseline vs $100 \mu \mathrm{M}$ picrotoxin, $p=0.52, n=4$ ), consistent with RAE (Fig. $1 F$ ) and Rayleigh analyses (Fig. 5C). Additionally, the long-period extreme also maintained precision and coherence at baseline levels $\left(F b x l 3^{A f h / A f h}\right.$ baseline vs $100 \mu \mathrm{M}$ KNK437, $\left.p=0.12, n=4\right)$, con-

(Figure legend continued.) degree of synchrony is indicated by the length of the vector in the center. $\boldsymbol{F}$, Summary synchrony data reported by mean vector length from individual Rayleigh analyses. Values are shown as mean \pm SEM for baseline (black), $100 \mu \mathrm{m}$ KNK437 (red), and 100 $\mu \mathrm{M}$ KNK437/1 $\mu \mathrm{M}$ TTX cotreatment (gray). G, Relative period range width from individual oscillators identified by SARFIA analysis in $\boldsymbol{A}$ and $\boldsymbol{D}$ expressed as a percentage of the overall cellular period. Values are shown as mean \pm SEM for baseline (black), treatment (red), and TTX cotreatment (gray). Period-altering treatment conditions are detailed below the bars $(+100$ $\mu \mathrm{m}$ picrotoxin or $+100 \mu \mathrm{m}$ KNK437), and genotypes are detailed above the bars (CK1 $\varepsilon^{\text {Tau/Tau }}$ $\times$ PER2::LUC or Fbx/3 $3^{A f h / A f h} \times$ PER2::LUC). $n$ values are detailed throughout the text. ${ }^{*} p<$ $0.05,{ }^{* *} p<0.01,{ }^{* * *} p<0.001,{ }^{* * * *} p<0.0001$. sistent with previous analyses (Figs. 1F, Fig. $5 F$ ). As expected, precision and coherence were lost when SCNs were treated with TTX at both period extremes $\left(C K 1 \varepsilon^{\text {Tau/Tau }}, 100 \mu \mathrm{M}\right.$ picrotoxin alone vs with $1 \mu \mathrm{M}$ TTX, $p<0.01, n=4 ; F b x l 3^{A f h / A f h}, 100 \mu \mathrm{M}$ KNK437 alone vs with $1 \mu \mathrm{M}$ TTX, $p<0.01, n=4$ ), illustrating the power of the network properties of the SCN when oscillating at these nonphysiological, extreme periods.

Temporal information in the SCN is not only encoded in time, but also through spatial waves of gene expression that flow across the network (Brancaccio et al., 2013). To determine whether pushing the SCN oscillation to extreme periods altered the informational content of the spatiotemporal wave, CoL analysis was applied to CCD recordings (Fig. 6A,C). The wave followed a highly repeatable orbit across the three cycles preceding pharmacological treatment and the first three cycles during pharmacological treatment. To assess whether the path length of the spatiotemporal wave differed between baseline and pharmacological treatment, a path index was calculated (Fig. 6B,D). This remained unaffected by pushing the $\mathrm{SCN}$ to extremely short (Fig. $6 B$; $C K 1 \varepsilon^{\text {Tau/Tau }}$ treated with $100 \mu \mathrm{M}$ picrotoxin, baseline path index vs treatment path index, $p=0.47, n=4$ ) or extremely long periods (Fig. 6D; Fbxl3 ${ }^{A f h / A f h}$ treated with $100 \mu \mathrm{M}$ KNK437, baseline path index vs treatment path index, $p=0.92, n=4)$. Thus, single-cell analysis of the network properties of the SCN under extreme periods indicates that the effects on PER2::LUC bioluminescent waveform are dependent solely on the state of the internal oscillator, not circuit-based rearrangements. These results also prove that the isolated $\mathrm{SCN}$ is a remarkable biological oscillator that can reversibly maintain coherent rhythms even when pushed well outside the physiological period range.

\section{Discussion}

In forcing the SCN to oscillate at extreme periods, we hypothesized three possible outcomes: (1) the network contains sufficient temporal elasticity to sustain extreme period oscillations; (2) the network is unable to sustain the oscillation and the slice becomes asynchronous, but cellular clock function is elastic and extreme cellular periods are retained; 3 ) the molecular oscillator is unable to sustain the oscillation and the slice becomes completely arrhythmic. Combined pharmacological and genetic manipulation of the SCN revealed that this structure forms a remarkable oscillator capable of maintaining coherent circadian rhythms of gene expression over an interval of between ca. 17 and $42 \mathrm{~h}$. In addition, not only are these oscillations coherently maintained at the level of the aggregate signal, but they are also maintained at the cell-autonomous and spatiotemporal network levels as well as being fully reversible. These experiments demonstrate that even when faced with the need to adapt to wildly inappropriate periods, the SCN can maintain oscillations at every level of timekeeping (Brancaccio et al., 2013; Brancaccio et al., 2014); i.e., the cell-autonomous clock and the network contain sufficient temporal elasticity to maintain extreme period oscillations.

For context, in a competent wild-type SCN explant, the periods expressed by individual oscillators range from $24.51 \pm 0.11$ to $25.18 \pm 0.13 \mathrm{~h}(n=4$; data not shown), an effective intra-SCN period range of $<1 \mathrm{~h}$. Between individual competent wild-type SCN explants, the period range of the aggregate signal is between $24.03 \pm 0.07$ and $25.26 \pm 0.19 \mathrm{~h}$ (calculated from baseline data; Fig. 1), an effective inter-SCN period range of $\sim 1.5 \mathrm{~h}$. These relatively small period ranges are imposed by tight interneuronal communication between oscillators (Yamaguchi et al., 2003). In other preparations that lack this degree of coupling between oscillators, the period range extends: for example, dissociated SCN 
A

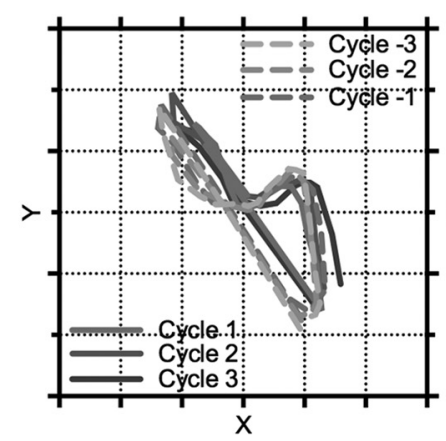
CK1 $\varepsilon^{\text {Tau/Tau }_{x} \text { PER2::LUC }}$

C

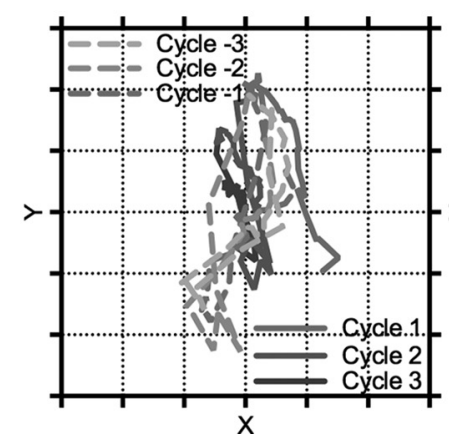

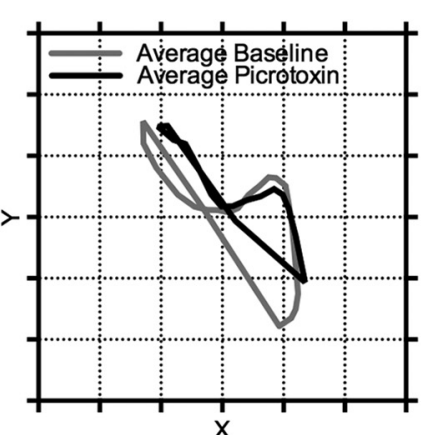

$\mathrm{x}$

\section{Baseline Picrotoxin}
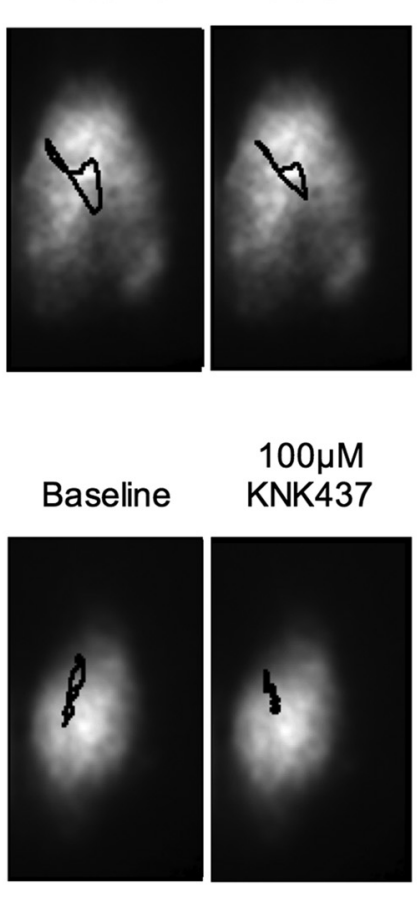

B

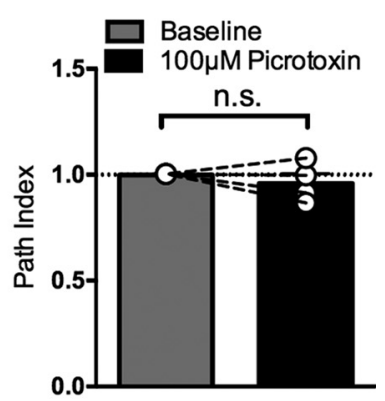

D

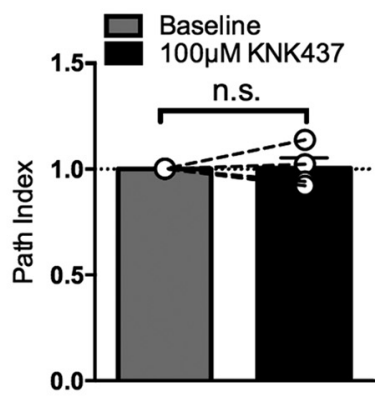

Figure 6. Network waveform properties of the SCN are unaffected by pushing period to short or long extremes. $A-D$, CoL analysis of CK1 $\varepsilon^{\text {Tau/Tau }} \times$ PER2::LUC treated with $100 \mu$ M picrotoxin $(\boldsymbol{A}, \boldsymbol{B})$ and Fbx/3 ${ }^{A f h / A f h} \times$ PER2::LUC treated with $100 \mu \mathrm{m}$ KNK437 $(\boldsymbol{C}, \boldsymbol{D})$. $\boldsymbol{A}$, Left, Representative path vectors of center of luminescence across the slice displaying individual paths for three cycles before (dashed lines, graded gray) and during $100 \mu \mathrm{m}$ picrotoxin application (solid lines, graded gray) and corresponding mean paths (right) showing baseline (gray) overlaid with $100 \mu \mathrm{m}$ picrotoxin (black). Right, Representative single images of one SCN overlaid with mean path vectors (black) for baseline (left) and $100 \mu \mathrm{M}$ picrotoxin (right). $\boldsymbol{B}$, Summary data showing mean path index for baseline (gray) and $100 \mu \mathrm{m}$ picrotoxin (black). Individual values are shown as hollow circles linked by dashed lines. $\boldsymbol{C}$, Left, Representative path vectors of center of luminescence across the slice displaying individual paths for three cycles before (dashed lines, graded gray) and during $100 \mu \mathrm{m}$ KNK437 application (solid lines, graded gray) and corresponding mean paths (right) showing baseline (gray) overlaid with $100 \mu$ M KNK437 (black). Right, Representative single images of one nucleus overlaid with mean path vectors (black) for baseline (left) and $100 \mu$ m KNK437 (right). D, Summary data showing mean path index for baseline (gray) and $100 \mu \mathrm{m}$ KNK437 (black). Individual values are shown as hollow circles linked by dashed lines. Bars indicate mean \pm SEM. $n$ values are as detailed in text.

neurons express periods between $\sim 22$ and $26 \mathrm{~h}$ (Herzog et al., 2004), while fibroblasts oscillate between 22 and $30 \mathrm{~h}$ (Welsh et al., 2004), effective period ranges of 4-8 h. None of these preparations, however, approach the ranges reported here either within $\left(C K 1 \varepsilon^{\text {Tau/Tau }}, 12.6 \mathrm{~h}\right.$; wild-type, $12.8 \mathrm{~h} ; \mathrm{Fb} x \mathrm{l} 3^{A f h / A f h}$, $21.7 \mathrm{~h})$ or between $(\sim 25 \mathrm{~h})$ genotypes, and, indeed, in a functional and coherent SCN network, this range is unprecedented.

The extreme period manipulations allowed us to reveal that the circadian oscillation of gene expression contains cryptic information. Our method of analyzing the waveform (Fig. 2) shows that the clock likely functions as a set of distinct stages, similarly to the cell cycle, with checkpoints and thresholds that need to be satisfied for the cycle to progress. This arrangement of clock progression has been hinted at before, where the clock moves through distinct transcriptional phases (Koike et al., 2012), although these phases refer to circadian output rather than direct progression of the clock per se. The FDA gives a parameter to clock analysis additional to phase, amplitude, and period of oscillations. The value of this analysis of waveform was revealed by treatment of the wild-type clock with the CK1 $\varepsilon$ inhibitor PF4800567 (Fig. 4), a treatment previously identified as ineffective (Meng et al., 2010), but the FDA revealed a subtle effect in the second half of the circadian cycle. This indicated that the CK1 $\varepsilon$ isoform activity is most sensitive to pharmacological manipulation during the interval at which PER2 degradation occurs, consistent with the previously proposed role of CK1 $1 \varepsilon$ (Lowrey et al., 2000; Meng et al., 2008; Maywood et al., 2014).
Aside from revealing phase ordering of the circadian oscillation of gene expression, these experiments show that there is a strong interaction between genetics and pharmacology to different degrees across phases. It is apparent from FDA-S analysis that pharmacological manipulation of period exploits the same phase sensitivities regardless of genotype, but the magnitude of these phase sensitivities depends on genotype. In this way, FDA-S provides a useful insight into critical phases of the oscillation where a genetic mutation either sensitizes or protects against pharmacological manipulation, revealing pharmacologically specific phase patterning of the oscillation. Mechanistically, this is obvious when looking at the $C K 1 \varepsilon^{T a u / T a u}$ and wild-type slices treated with the CK1 $\varepsilon$ specific inhibitor PF-4800567, where specific inhibition of CK1 $\varepsilon$ highlights a significant genotype by pharmacology interaction over the first three quarters of the cycle that is attenuated toward the end of the cycle compared to the wild-type condition. This indicates critical internal phases where CK1 $\varepsilon$ alters period length in the $C K 1 \varepsilon^{\text {Tau/Tau }}$ mutation through inappropriately phased activity.

These analyses have been applied to the PER2::LUC waveform, which reports levels of the PER2 protein and therefore acts as a translational reporter (Yoo et al., 2004). In this way, the FDA only reports changes in PER2 dynamics and does not report causality. The changes here are likely driven by direct perturbation of other axes of the circadian oscillation that manifest as changes to the waveform of the PER2::LUC reporter. These range from direct perturbation of the PER2::LUC reporter through CK1 
manipulation (CK1 $\varepsilon^{\text {Tau/Tau }}, \mathrm{PF}-670462$, and PF-4800567) or through indirect perturbation of the PER2::LUC reporter through the CRY-mediated axis of timekeeping ( Fbxl $3^{\text {Afh/Afh }}$ ). Alternative approaches have used other circadian reporters, for example $\left[\mathrm{Ca}^{2+}\right]_{\mathrm{i}}$ imaging (Brancaccio et al., 2013) or transcriptional luciferase reporters (Maywood et al., 2013; Parsons et al., 2015). Applying these analyses to different reporters may provide more insight into the phase arrangement of the circadian oscillation and how these different axes are interwoven. It is likely that the phase arrangement of such reporters differs from PER2::LUC, as some of these reporters have very obvious waveform differences, e.g., the narrow peak and wide trough of $\left[\mathrm{Ca}^{2+}\right]_{i}$ and AT-luciferase reporters (Brancaccio et al., 2013; Parsons et al., 2015). Analysis of the waveform, for example, reveals that the peak rate of PER2 accumulation occurs between approximately circadian time 6 and circadian time 7.2 (normalized period, 0.25 0.3; Fig. 2). This coincides with the previously established peaks of calcium (Brancaccio et al., 2013) and spontaneous firing rate (Atkinson et al., 2011; Colwell, 2011). This is consistent with cAMP/Ca ${ }^{2+}$-response elements in the promoter of Per genes (Obrietan et al., 1999; Tischkau et al., 2003; O’Neill and Reddy, 2012) and illustrates one of the ways that this sort of analysis of gene expression dynamics can be used to further understand how different axes of timekeeping interact. Additionally, combination of FDA with real-time gene expression imaging in various tissues of freely moving animals (Ono et al., 2015a;b; Hamada et al., 2016) could allow pharmacologically sensitive phases of the circadian waveform to be aligned with behavioral data.

Finally, the circadian oscillator in the SCN provides a valuable natural tool to study the function of biological oscillators at both the cell-autonomous and network levels, and for this reason it has been the focus of mathematical modeling to explain these processes (Fuhr et al., 2015). Although these models have been used to great effect in combination with experimental data to study other properties of the SCN, such as intercellular coupling (DeWoskin et al., 2015; Myung et al., 2015) and phase entrainment (Bordyugov et al., 2015), few have modeled different period scales outside of $24 \mathrm{~h}$. In this investigation, we present data from nine different genotype-pharmacology combinations that provide data on nine different period conditions that the SCN is able to sustain, and which reveal differential kinetics due to genotypepharmacology interactions. Although these conditions are well outside the physiological range that the SCN would need to sustain, they are a product of SCN timekeeping in response to specific perturbations, and therefore represent behaviors of the SCN that bona fide models should be able to interrogate, replicate, and explain.

Furthermore, many biological processes exhibit oscillatory behavior on a broad range of timescales, for example, hormone cycles, dynamic calcium oscillations, and metabolic processes (Kim et al., 2010). Extending the analysis of waveforms beyond the circadian system may reveal underlying mechanisms of regulation to these oscillations. This could be especially interesting in considering how spontaneous slow-wave calcium oscillations propagate across the whole brain, what their kinetics are, and how oscillations arising from different brain areas interact at the phase-specific level. Measurements of such types of oscillation have not only been limited to normal function (Stroh et al., 2013), but have proved useful in developing an understanding of a range of different neuronal states, from neuronal maturation during development (Garaschuk et al., 2000) to disruption of long-range signals during neurodegeneration (Busche et al., 2015). Complementing these imaging tools with measurements of the kinetics of the calcium signal through circadian time could provide important information as to how networks interact across the whole brain and how these interactions are disrupted during pathogenesis.

\section{References}

Atkinson SE, Maywood ES, Chesham JE, Wozny C, Colwell CS, Hastings MH, Williams SR (2011) Cyclic AMP signaling control of action potential firing rate and molecular circadian pacemaking in the suprachiasmatic nucleus. J Biol Rhythms 26:210-220. CrossRef Medline

Bordyugov G, Abraham U, Granada A, Rose P, Imkeller K, Kramer A, Herzel H (2015) Tuning the phase of circadian entrainment. J R Soc Interface 12:20150282. CrossRef Medline

Brancaccio M, Maywood ES, Chesham JE, Loudon AS, Hastings MH (2013) A Gq-Ca2+ axis controls circuit-level encoding of circadian time in the suprachiasmatic nucleus. Neuron 78:714-728. CrossRef Medline

Brancaccio M, Enoki R, Mazuski CN, Jones J, Evans JA, Azzi A (2014) Network-mediated encoding of circadian time: the suprachiasmatic nucleus (SCN) from genes to neurons to circuits, and back. J Neurosci 34:15192-15199. CrossRef Medline

Buhr ED, Yoo SH, Takahashi JS (2010) Temperature as a universal resetting cue for mammalian circadian oscillators. Science 330:379-385. CrossRef Medline

Busche MA, Kekuš M, Adelsberger H, Noda T, Förstl H, Nelken I, Konnerth A (2015) Rescue of long-range circuit dysfunction in Alzheimer's disease models. Nat Neurosci 18:1623-1630. CrossRef Medline

Colwell CS (2011) Linking neural activity and molecular oscillations in the SCN. Nat Rev Neurosci 12:553-569. CrossRef Medline

DeWoskin D, Myung J, Belle MD, Piggins HD, Takumi T, Forger DB (2015) Distinct roles for GABA across multiple timescales in mammalian circadian timekeeping. Proc Natl Acad Sci U S A 112:E3911-E3919. CrossRef Medline

Dorostkar MM, Dreosti E, Odermatt B, Lagnado L (2010) Computational processing of optical measurements of neuronal and synaptic activity in networks. J Neurosci Methods 188:141-150. CrossRef Medline

Freeman GM Jr, Nakajima M, Ueda HR, Herzog ED (2013) Picrotoxin dramatically speeds the mammalian circadian clock independent of Cys-loop receptors. J Neurophysiol 110:103-108. CrossRef Medline

Fuhr L, Abreu M, Pett P, Relógio A (2015) Circadian systems biology: when time matters. Comput Struct Biotechnol J 13:417-426. CrossRef Medline

Garaschuk O, Linn J, Eilers J, Konnerth A (2000) Large-scale oscillatory calcium waves in the immature cortex. Nat Neurosci 3:452-459. CrossRef Medline

Godinho SI, Maywood ES, Shaw L, Tucci V, Barnard AR, Busino L, Pagano M, Kendall R, Quwailid MM, Romero MR, O'Neill J, Chesham JE, Brooker D, Lalanne Z, Hastings MH, Nolan PM (2007) The after-hours mutant reveals a role for Fbxl3 in determining mammalian circadian period. Science 316:897-900. CrossRef Medline

Hamada T, Sutherland K, Ishikawa M, Miyamoto N, Honma S, Shirato H, Honma K (2016) In vivo imaging of clock gene expression in multiple tissues of freely moving mice. Nat Commun 7:11705. CrossRef Medline

Hastings M, O'Neill JS, Maywood ES (2007) Circadian clocks: regulators of endocrine and metabolic rhythms. J Endocrinol 195:187-198. CrossRef Medline

Hastings MH, Reddy AB, McMahon DG, Maywood ES (2005) Analysis of circadian mechanisms in the suprachiasmatic nucleus by transgenesis and biolistic transfection. Methods Enzymol 393:579-592. CrossRef Medline

Hastings MH, Brancaccio M, Maywood ES (2014) Circadian pacemaking in cells and circuits of the suprachiasmatic nucleus. J Neuroendocrinol 26: 2-10. CrossRef Medline

Herzog ED, Aton SJ, Numano R, Sakaki Y, Tei H (2004) Temporal precision in the mammalian circadian system: a reliable clock from less reliable neurons. J Biol Rhythms 19:35-46. CrossRef Medline

Kim JR, Shin D, Jung SH, Heslop-Harrison P, Cho KH (2010) A design principle underlying the synchronization of oscillations in cellular systems. J Cell Sci 123:537-543. CrossRef Medline

Koike N, Yoo SH, Huang HC, Kumar V, Lee C, Kim TK, Takahashi JS (2012) Transcriptional architecture and chromatin landscape of the core circadian clock in mammals. Science 338:349-354. CrossRef Medline

Lee H, Chen R, Lee Y, Yoo S, Lee C (2009) Essential roles of CKIdelta and CKIepsilon in the mammalian circadian clock. Proc Natl Acad Sci U S A 106:21359-21364. CrossRef Medline 
Liu AC, Welsh DK, Ko CH, Tran HG, Zhang EE, Priest AA, Buhr ED, Singer O, Meeker K, Verma IM, Doyle FJ 3rd, Takahashi JS, Kay SA (2007) Intercellular coupling confers robustness against mutations in the SCN circadian clock network. Cell 129:605-616. CrossRef Medline

Lowrey PL, Shimomura K, Antoch MP, Yamazaki S, Zemenides PD, Ralph MR, Menaker M, Takahashi JS (2000) Positional syntenic cloning and functional characterization of the mammalian circadian mutation tau. Science 288:483-492. CrossRef Medline

Maywood ES, Reddy AB, Wong GK, O’Neill JS, O’Brien JA, McMahon DG, Harmar AJ, Okamura H, Hastings MH (2006) Synchronization and maintenance of timekeeping in suprachiasmatic circadian clock cells by neuropeptidergic signaling. Curr Biol 16:599-605. CrossRef Medline

Maywood ES, Chesham JE, O’Brien JA, Hastings MH (2011a) A diversity of paracrine signals sustains molecular circadian cycling in suprachiasmatic nucleus circuits. Proc Natl Acad Sci U S A 108:14306-14311. CrossRef Medline

Maywood ES, Chesham JE, Meng QJ, Nolan PM, Loudon AS, Hastings MH (2011b) Tuning the period of the mammalian circadian clock: additive and independent effects of CK1epsilonTau and Fbxl3Afh mutations on mouse circadian behavior and molecular pacemaking. J Neurosci 31: 1539-1544. CrossRef Medline

Maywood ES, Drynan L, Chesham JE, Edwards MD, Dardente H, Fustin JM, Hazlerigg DG, O'Neill JS, Codner GF, Smyllie NJ, Brancaccio M, Hastings MH (2013) Analysis of core circadian feedback loop in suprachiasmatic nucleus of mCryl-luc transgenic reporter mouse. Proc Natl Acad Sci U S A 110:9547-9552. CrossRef Medline

Maywood ES, Chesham JE, Smyllie NJ, Hastings MH (2014) The Tau mutation of casein kinase lepsilon sets the period of the mammalian pacemaker via regulation of Period 1 or Period 2 clock proteins. J Biol Rhythms 29:110-118. CrossRef Medline

Meng QJ, Logunova L, Maywood ES, Gallego M, Lebiecki J, Brown TM, Sládek M, Semikhodskii AS, Glossop NR, Piggins HD, Chesham JE, Bechtold DA, Yoo SH, Takahashi JS, Virshup DM, Boot-Handford RP, Hastings MH, Loudon AS (2008) Setting clock speed in mammals: the CK1 epsilon tau mutation in mice accelerates circadian pacemakers by selectively destabilizing PERIOD proteins. Neuron 58:78-88. CrossRef Medline

Meng QJ, Maywood ES, Bechtold DA, Lu WQ, Li J, Gibbs JE, Dupré SM, Chesham JE, Rajamohan F, Knafels J, Sneed B, Zawadzke LE, Ohren JF, Walton KM, Wager TT, Hastings MH, Loudon AS (2010) Entrainment of disrupted circadian behavior through inhibition of casein kinase 1 (CK1) enzymes. Proc Natl Acad Sci U S A 107:15240-15245. CrossRef Medline

Moore A, Zielinski T, Millar AJ (2014) Online period estimation and determination of rhythmicity in circadian data, using the BioDare data infrastructure. Methods Mol Biol 1158:13-44. CrossRef Medline

Myung J, Hong S, DeWoskin D, De Schutter E, Forger DB, Takumi T (2015) GABA-mediated repulsive coupling between circadian clock neurons in the SCN encodes seasonal time. Proc Natl Acad Sci U S A 112:E3920E3929. CrossRef Medline

Obrietan K, Impey S, Smith D, Athos J, Storm DR (1999) Circadian regulation of cAMP response element-mediated gene expression in the suprachiasmatic nuclei. J Biol Chem 274:17748-17756. CrossRef Medline

O'Neill JS, Hastings MH (2008) Increased coherence of circadian rhythms in mature fibroblast cultures. J Biol Rhythms 23:483-488. CrossRef Medline

O'Neill JS, Reddy AB (2012) The essential role of cAMP/Ca2 + signalling in mammalian circadian timekeeping. Biochem Soc Trans 40:44-50. CrossRef Medline
Ono D, Honma S, Honma K (2015a) Circadian PER2::LUC rhythms in the olfactory bulb of freely moving mice depend on the suprachiasmatic nucleus but not on behaviour rhythms. Eur J Neurosci 42:3128-3137. CrossRef Medline

Ono D, Honma K, Honma S (2015b) Circadian and ultradian rhythms of clock gene expression in the suprachiasmatic nucleus of freely moving mice. Sci Rep 5:12310. CrossRef Medline

Parsons MJ, Brancaccio M, Sethi S, Maywood ES, Satija R, Edwards JK, Jagannath A, Couch Y, Finelli MJ, Smyllie NJ, Esapa C, Butler R, Barnard AR, Chesham JE, Saito S, Joynson G, Wells S, Foster RG, Oliver PL, Simon MM, et al. (2015) The regulatory factor ZFHX3 modifies circadian function in SCN via an AT motif-driven axis. Cell 162:607-621. CrossRef Medline

Pilorz V, Cunningham PS, Jackson A, West AC, Wager TT, Loudon AS, Bechtold DA (2014) A novel mechanism controlling resetting speed of the circadian clock to environmental stimuli. Curr Biol 24:766-773. CrossRef Medline

Ralph MR, Menaker M (1988) A mutation of the circadian system in golden hamsters. Science 241:1225-1227. CrossRef Medline

Schindelin J, Arganda-Carreras I, Frise E, Kaynig V, Longair M, Pietzsch T, Preibisch S, Rueden C, Saalfeld S, Schmid B, Tinevez JY, White DJ, Hartenstein V, Eliceiri K, Tomancak P, Cardona A (2012) Fiji: an opensource platform for biological-image analysis. Nat Methods 9:676-682. CrossRef Medline

Stroh A, Adelsberger H, Groh A, Rühlmann C, Fischer S, Schierloh A, Deisseroth K, Konnerth A (2013) Making waves: initiation and propagation of corticothalamic Ca2+ waves in vivo. Neuron 77:1136-1150. CrossRef Medline

Tischkau SA, Mitchell JW, Tyan SH, Buchanan GF, Gillette MU (2003) $\mathrm{Ca} 2+/ \mathrm{cAMP}$ response element-binding protein (CREB)-dependent activation of Per1 is required for light-induced signaling in the suprachiasmatic nucleus circadian clock. J Biol Chem 278:718-723. CrossRef Medline

Walton KM, Fisher K, Rubitski D, Marconi M, Meng QJ, Sládek M, Adams J, Bass M, Chandrasekaran R, Butler T, Griffor M, Rajamohan F, Serpa M, Chen Y, Claffey M, Hastings M, Loudon A, Maywood E, Ohren J, Doran A, et al. (2009) Selective inhibition of casein kinase 1 epsilon minimally alters circadian clock period. J Pharmacol Exp Ther 330:430-439. CrossRef Medline

Welsh DK, Yoo SH, Liu AC, Takahashi JS, Kay SA (2004) Bioluminescence imaging of individual fibroblasts reveals persistent, independently phased circadian rhythms of clock gene expression. Curr Biol 14:2289-2295. CrossRef Medline

Yamaguchi S, Isejima H, Matsuo T, Okura R, Yagita K, Kobayashi M, Okamura H (2003) Synchronization of cellular clocks in the suprachiasmatic nucleus. Science 302:1408-1412. CrossRef Medline

Yamazaki S, Straume M, Tei H, Sakaki Y, Menaker M, Block GD (2002) Effects of aging on central and peripheral mammalian clocks. Proc Natl Acad Sci U S A 99:10801-10806. CrossRef Medline

Yoo SH, Yamazaki S, Lowrey PL, Shimomura K, Ko CH, Buhr ED, Siepka SM, Hong HK, Oh WJ, Yoo OJ, Menaker M, Takahashi JS (2004) PERIOD2::LUCIFERASE real-time reporting of circadian dynamics reveals persistent circadian oscillations in mouse peripheral tissues. Proc Natl Acad Sci U S A 101:5339-5346. CrossRef Medline

Zielinski T, Moore AM, Troup E, Halliday KJ, Millar AJ (2014) Strengths and limitations of period estimation methods for circadian data. PLoS One 9:e96462. CrossRef Medline 